\title{
Adaptive ecological processes and metabolic independence drive microbial colonization and resilience in the human gut
}

Andrea R. Watson ${ }^{1,2}$, Jessika Füssel ${ }^{1}$, Iva Veseli ${ }^{3}$, Johanna Zaal DeLongchamp ${ }^{4}$, 5 Marisela Silva ${ }^{4}$, Florian Trigodet ${ }^{1}$, Karen Lolans ${ }^{1}$, Alon Shaiber ${ }^{4}$, Emily Fogarty ${ }^{1,2}$, Joseph M. Runde ${ }^{5}$, Christopher Quince ${ }^{6}$, Michael K. Yu , Arda Söylev ${ }^{8}$, Hilary G. Morrison ${ }^{9}$, Sonny T.M. Lee ${ }^{1}$, Dina Kao ${ }^{9}$, David T. Rubin ${ }^{1}$, Bana Jabri' ${ }^{1}$, Thomas Louie ${ }^{3}$, A. Murat Eren $^{1,2,4,10, *}$

${ }^{1}$ Department of Medicine, The University of Chicago, Chicago, IL 60637, USA

${ }^{2}$ Committee on Microbiology, The University of Chicago, Chicago, IL 60637, USA

${ }^{3}$ Biophysical Sciences Program, The University of Chicago, Chicago, IL 60637, USA

${ }^{4}$ Department of Medicine, The University of Calgary, Calgary, AB T2N 1N4, Canada

${ }^{5}$ Department of Pediatrics, Lurie Children's Hospital of Chicago, Chicago, IL 60611, USA

${ }^{6}$ Organisms and Ecosystems, Earlham Institute, Norwich, Norwich, NR4 7UZ, United Kingdom

${ }^{7}$ Toyota Technological Institute at Chicago, Chicago, IL 60637, USA

${ }^{8}$ Department of Computer Engineering, Konya Food and Agriculture University, Konya, Turkey

${ }^{9}$ Department of Medicine, University of Alberta, Edmonton, AB T6G 2G3, Canada

${ }^{10}$ Josephine Bay Paul Center, Marine Biological Laboratory, Woods Hole, MA 02543, USA

20 * Corresponding author: meren@uchicago.edu

Running Title Drivers of human gut colonization

Keywords fecal microbiota transplantation; human gut microbiome; microbial colonization; microbial metabolism; metabolic competency. 


\section{Abstract}

A detailed understanding of human gut microbial ecology is essential to engineer effective microbial therapeutics and to model microbial community assembly in health and disease. However, establishing generalizable insights into the functional determinants of microbial fitness in the gut has been a formidable challenge. Here we employ fecal microbiota

30 transplantation (FMT) as an in natura experimental model to identify determinants of microbial colonization and resilience. Our findings reveal adaptive ecological processes that favor high-fitness populations with higher metabolic competence as the main driver of microbial colonization outcomes after FMT. We further show that while healthy individuals harbor both low-fitness and high-fitness populations, individuals with

35 inflammatory bowel disease are typically depleted of low-fitness populations. These results offer a model to explain why common yet typically rare members of healthy guts can dominate under inflammatory conditions without any need for them to be causally associated with, or contribute to, such disease states. 


\section{Introduction}

The human gut microbiome is associated with a wide range of diseases and disorders (Almeida et al., 2020; Durack and Lynch, 2019; Lynch and Pedersen, 2016). However, mechanistic underpinnings of these associations have been difficult to resolve in part due to the diversity of human lifestyles (David et al., 2014) and the limited utility of model

45 systems to make robust causal inferences for microbially mediated human diseases (Walter et al., 2020).

Inflammatory bowel disease (IBD), a group of increasingly common intestinal disorders that cause inflammation of the gastrointestinal tract (Baumgart and Carding, 2007), has been a model to study human diseases associated with the gut microbiota (Schirmer et

50 al., 2019). The pathogenesis of IBD is attributed in part to the gut microbiome (Plichta et al., 2019), yet the microbial ecology of IBD-associated dysbiosis remains a puzzle. Despite marked changes in gut microbial community composition in IBD (Joossens et al., 2011; Ott et al., 2004; Sokol and Seksik, 2010), the microbiota associated with the disease lacks traditional pathogens (Chow et al., 2011), and microbes that are found in

55 IBD typically also occur in healthy individuals (Clooney et al., 2021), which complicates the search for robust functional or taxonomic markers of health and disease states (LloydPrice et al., 2019). One of the hallmarks of IBD is reduced microbial diversity during episodes of inflammation, when the gut environment is often dominated by microbes that typically occur in lower abundances prior to inflammation (Vineis et al., 2016). The sudden

60 increase in the relative abundance of microbes that are common to healthy individuals suggests that the harsh conditions of IBD likely act as an ecological filter that prevents the persistence of low-fitness populations. Yet, in the absence of a complete understanding of the functional drivers of microbial colonization in this habitat, critical insights into the metabolic requirements of survival in IBD remains elusive.

65 Understanding the determinants of microbial colonization has been one of the fundamental aims of gut microbial ecology (Costello et al., 2012; Messer et al., 2017). To overcome the difficulties of conducting well-controlled studies with humans, researchers have studied the determinants of microbial colonization of the gut in model systems, such 
as germ-free mice conventionalized with individual taxa (Lee et al., 2013) or a consortium

70 of human microbial isolates (Feng et al., 2020). Despite their utility for hypothesis testing, simpler models do not capture the complex ecological interactions fostered by natural systems and thus the insights they yield do not always translate to human gut microbial ecology (Finucane et al., 2014; Ley et al., 2006). Between the extremes of well-controlled but simple mouse models and complex yet uncontrolled human populations, there exists

75 a middleground that provides a window into the microbial ecology of complex human systems through a controlled perturbation: human fecal microbiota transplantation (FMT), the transfer of stool from a donor into a recipient's gastrointestinal tract (Eiseman et al., 1958).

FMT complements laboratory models of environmental perturbation by colliding two

80 distinct microbial ecosystems, and thus offers a powerful framework to study fundamental questions of microbial ecology, including the determinants of microbial succession and resilience (Schmidt et al., 2018). Here we use FMT as an in natura experimental model to investigate the ecological and functional determinants of successful microbial colonization of the human gut at the level of individual populations. Our findings suggest

85 that adaptive ecological forces are key drivers of colonization outcomes after FMT, reveal taxonomy-independent metabolic determinants of fitness in the human gut, and demonstrate that similar ecological principles determine resilience of microbes in stressful and inflammatory conditions.

\section{Results and Discussion}

90 Our study includes 109 gut metagenomes (Supplementary Table 1) from two healthy FMT donors (A and $B$ ) and 10 FMT recipients (five recipients per donor) who had multiply recurrent Clostridium difficile infection (CDI) and received vancomycin for a minimum of 10 days to attain resolution of diarrheal illness prior to FMT. On the last day of vancomycin treatment, a baseline fecal sample was collected from each recipient, and their bowel

95 contents were evacuated immediately prior to FMT. Recipients did not take any antibiotics on the day of transplant, or during the post-FMT sampling period (Supplementary Figure 1). We also collected 24 Donor A samples over a period of 636 days and 15 Donor B 
samples over a period of 532 days to establish an understanding of the long-term microbial population dynamics within each donor microbiota. We also collected 5 to 9 100 samples from each recipient up to 336 days post-FMT. Deep sequencing of donor and recipient metagenomes using Illumina paired-end $(2 \times 150)$ technology resulted in a total of 7.7 billion sequences with an average of 71 million reads per metagenome (Figure 1, Supplementary Table 1, Supplementary Table 2). We employed genome-resolved metagenomics, pangenomics, and microbial population genetics for an in-depth

105 characterization of donor and recipient gut microbiota using these data, and we leveraged publicly available gut metagenomes to benchmark our observations.

\section{Many but not all donor microbes colonized recipients and persisted long-term}

We first characterized the taxonomic composition of each donor and recipient sample by

110 aligning metagenomic short reads to reference genomes in the NCBI's RefSeq database (Supplementary Table 2). The phylum-level microbial community composition of both donors reflected those observed in healthy individuals in North America (Human Microbiome Project Consortium, 2012): a large representation of Firmicutes and Bacteroidetes, and other taxa with relatively lower relative abundances, including

115 Actinobacteria, Verrucomicrobia, and Proteobacteria (Figure 1, Supplementary Table 2). In contrast, the vast majority of the recipient pre-FMT samples were dominated by Proteobacteria, a phylum that typically undergoes a drastic expansion in individuals treated with vancomycin (Isaac et al., 2017). After the FMT, we observed a dramatic shift in recipient taxonomic profiles (Supplementary Table 2, Supplementary Figure 2), a 120 widely documented hallmark of this procedure (Grehan et al., 2010; Khoruts et al., 2010; Shahinas et al., 2012). Nearly all recipient samples post-FMT were dominated by Bacteroidetes and Firmicutes as well as Actinobacteria and Verrucomicrobia in lower abundances, resembling qualitatively, but not quantitatively, the taxonomic profiles of their donors (Supplementary Table 2). For example, even though the median relative

125 abundance of Bacteroidetes populations were $5 \%$ and $17 \%$ in donors $A$ and $B$, their relative abundance in recipients post-FMT increased to $33 \%$ and $45 \%$, respectively 
(Figure 1, Supplementary Table 2). A single genus, Bacteroides, made up $76 \%$ and $82 \%$ of the Bacteroidetes populations in the recipients of Donor $A$ and $B$, respectively (Supplementary Table 2). The success of the donor Bacteroides populations in recipients

130 upon FMT is not surprising given the ubiquity of this genus across human populations throughout the globe (Wexler and Goodman, 2017) and the ability of its members to survive substantial levels of stress (Swidsinski et al., 2005; Vineis et al., 2016). This result suggests that FMT outcomes in our dataset are unlikely random, and the study design and resulting dataset offers a framework to study ecological principles of the human gut

135 microbiome.

Next, we assembled short metagenomic reads into contiguous segments of DNA (contigs). Co-assemblies of 24 Donor A and 15 Donor B metagenomes independently resulted in 53,891 and 54,311 contigs that were longer than 2,500 nucleotides, and described 0.70 and 0.79 million genes occurring in 179 and 248 genomes, as estimated

140 by the mode of the frequency of bacterial single-copy core genes (Supplementary Table 2). One way to characterize how well a given assembly describes the DNA content of a given metagenome is to calculate the percentage of reads it recruits from the metagenome through read mapping. Donor contigs recruited on average $80.8 \%$ of metagenomic reads from donor metagenomes. In contrast, they recruited $43.4 \%$ of reads

145 on average from pre-FMT recipient metagenomes. This number increased to $80.2 \%$ for recipient metagenomes post-FMT (Figure 1), and the donor contigs continued to represent $76.8 \%$ of the recipient metagenomes on average even after a year post-FMT (Supplementary Table 2). These read recruitment results suggest that members of the donor microbiota successfully established in recipient guts upon FMT and largely

150 persisted until the end of the sampling period.

Compared to metagenomic short reads, assembled contigs provide a larger genetic context to study microbial metagenomes. However, a sole focus on contigs may yield misleading results (Kowarsky et al., 2017) that can be ameliorated by reconstructing microbial genomes from metagenomic assemblies (Chen et al., 2020). We reconstructed

155 genomes from co-assembled donor metagenomes by grouping contigs into putative bins based on sequence composition and differential coverage signal as previously described 
(Lee et al., 2017; Sharon et al., 2013). We retained bins that were at least $70 \%$ complete and had no more than $10 \%$ redundancy as predicted by bacterial single-copy core genes (Bowers et al., 2017; Chen et al., 2020) and manually refined them to improve their quality 160 following previously described approaches (Delmont et al., 2018; Shaiber et al., 2020). Our binning resulted in a final list of 128 metagenome-assembled genomes (MAGs) for Donor $A$ and 183 MAGs for Donor B that included members of Firmicutes $(n=265)$, Bacteroidetes $(n=20)$, Actinobacteria $(n=14)$, Proteobacteria $(n=7)$, Verrucomicrobia $(n=2)$, Cyanobacteria $(n=2)$, and Patescibacteria $(n=1)$ (Supplementary Table 3). The

165 taxonomy of donor-derived genomes largely reflected the taxonomic composition of donor metagenomes as predicted by short reads (Figure 1, Supplementary Table 2, Supplementary Table 3). While only 20 genomes (mostly of Bacteroides and Alistipes) explained the entirety of the Bacteroidetes group, we recovered 265 MAGs that represented lower abundance but diverse populations of Firmicutes (Figure 1,

170 Supplementary Table 2, Supplementary Table 3). We found no difference between the delivery method of FMT for the recipients of donor A, where, on average $45 \%$ and $43 \%$ of donor genomes emerged in recipients who received donor stool through colonoscopy $(n=3)$ versus pills $(n=2)$, respectively. However, there was an increase in the efficiency of pills for donor B, where on average $25 \%$ and $54 \%$ of donor genomes emerged in 175 recipients who received donor stool through colonoscopy $(n=2)$ versus pill $(n=3)$ (Supplementary Figure 3).

Reconstructing genomes gave us access to microbial populations in metagenomes through metagenomic read recruitment strategies and enabled us to characterize (1) population-level microbial colonization dynamics before and after FMT using donor and 180 recipient metagenomes and (2) the distribution of each donor population across geographically distributed humans using 1,984 publicly available human gut metagenomes (Supplementary Table 4). As expected, we detected each donor population in at least one donor metagenome (see Methods for 'detection' criteria). Yet, only $16 \%$ of Donor A populations were detected in every Donor A sample, and only $44 \%$ 185 of Donor B MAGs were detected in every Donor B sample (Figure 1, Supplementary Table 3 ), in agreement with the previously documented dynamism of gut microbial community composition over time (David et al., 2014). A marked increase in the detection of donor 
populations in recipients after FMT echoed the general pattern of transfer suggested by the short-read taxonomy (Figure 1): while only $38 \%$ of Donor A and $54 \%$ of Donor B

190 populations were detected in at least one recipient pre-FMT, these percentages increased to $96 \%$ and $96 \%$ post-FMT (Supplementary Table 3). Not every donor population colonized each recipient, but colonization events did not appear to be random: while some donor populations colonized all recipients, others colonized none (Figure 1), providing us with an opportunity to resolve colonization events and quantify colonization success for 195 each donor population in our dataset. 


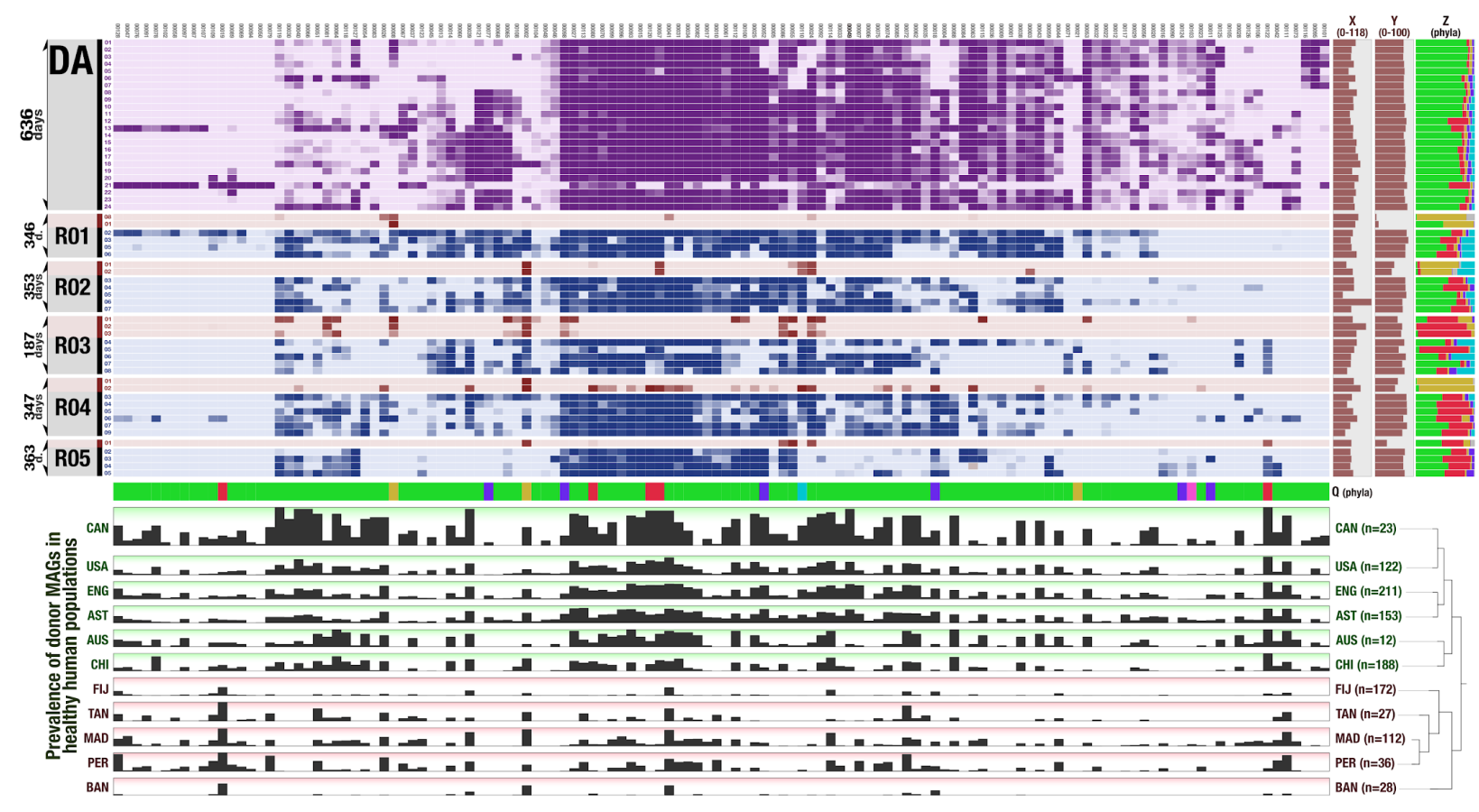

X: Number of metagenomic short reads per sample (millions) $\quad Y$ : \% of metagenomic reads recruited by donor MAGs $\quad$ Z: Phylum-level short read taxonomy per sample $\quad$ Q: Phylum-level genome taxonomy per donor MAG

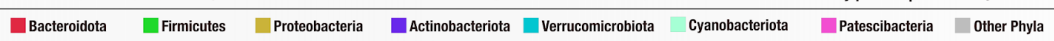
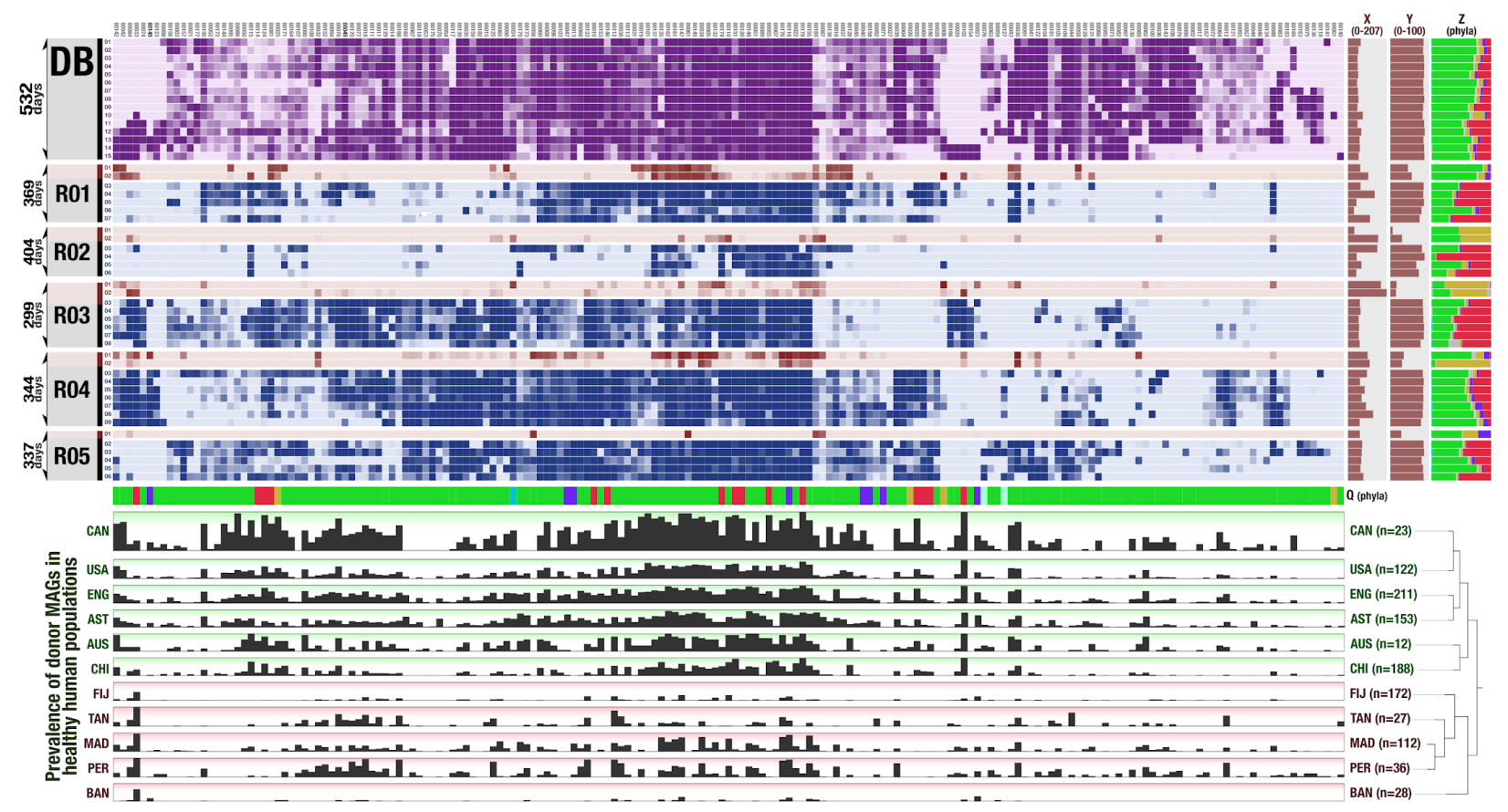

Figure 1. FMT Donor genomes across recipients and publicly available gut metagenomes. In both heat maps each column represents a donor genome and each row represents a metagenome, and each data point represents the detection of a given genome in a given metagenome. Purple rows represent donor metagenomes which cover 636 200 days for Donor A and 532 days for Donor B. Each recipient metagenome is colored red for pre-FMT samples and blue for post-FMT samples. The three rightmost columns display for each metagenome $(X)$ the number of metagenomic short reads in millions, $(\mathrm{Y})$ the percent of metagenomic short reads recruited by genomes, and $(\mathrm{Z})$ the taxonomic 
composition of metagenomes (based on metagenomic short reads) at the phylum level. The row $Q$ provides the phylumlevel taxonomy for each donor genome. Finally, the 11 bottom rows under each heat map show the fraction of healthy adult metagenomes from 11 different countries in which a given donor genome is detected (if a genome is detected in every individual from a country it is represented with a full bar). The dendrograms on the right-hand side of these layers organize countries based on the detection patterns of genomes (Euclidean distance and Ward clustering). Red and green shades represent the two main clusters that emerge from this analysis, where green layers are industrialized countries in which donor genomes are highly prevalent and red layers are less industrialized countries where the

210 prevalence of donor genomes is low.

Resolving colonization events accurately is a challenging task as multiple factors may influence the ability to determine colonization outcomes unambiguously. These factors include (1) the inability to detect low-abundance populations, (2) inaccurate characterization of transient populations observed immediately after FMT as successful

215 colonization events, (3) the reliance on relative abundance of populations to define colonization events when abundance estimates from stool do not always reflect the abundance of organisms in the GI tract (Sheth et al., 2019; Yasuda et al., 2015), and (4) the failure to distinguish between colonization by a donor population or emergence of a pre-FMT recipient population after FMT (where a low-abundance recipient population that

220 is closely related to one or more donor populations becomes abundant after FMT and is mistaken as a bona fide colonization event). To mitigate these factors, we have (1) employed deep-sequencing of our metagenomes which averaged 71 million reads per sample, (2) implemented a longitudinal sampling strategy, that spanned 376 days on average, to observe donor populations in our recipients long after the FMT, (3) leveraged

225 a 'detection' metric to define colonization events by presence/absence of populations rather than abundance, and (4) employed microbial population genetics to identify and resolve origins of subpopulations. We also developed an analytical approach (Supplementary Figure 4) to determine whether a given donor population has colonized a given recipient based on the detection of donor subpopulations in the transplant sample, 230 in the recipient pre-FMT, and in the recipient post-FMT (see Materials and Methods, Supplementary Table 5). To determine colonization outcomes, we analyzed 640 genome/recipient pairs for Donor A (128 donor genomes in 5 recipients) and identified 99 successful colonization events, 38 failed colonization events, and 503 ambiguous colonization events (Supplementary Table 6). For Donor B, we analyzed 915 235 genome/recipient pairs (183 donor genomes in 5 recipients) and identified 106 successful 
colonization events, 109 failed colonization events, and 700 ambiguous colonization events (Supplementary Table 6). Our stringent criteria (see Materials and Methods, Supplementary Figure 4) classified the vast majority of all genome/recipient pairs as ambiguous colonization events. Nevertheless, due to the relatively large number of donor

240 MAGs and FMT recipients in our study, we were left with 352 MAG/recipient pairs with unambiguous phenotypes for downstream analyses.

\section{Adaptive ecological forces are the primary drivers of microbial colonization}

The ability of a microbial population to colonize and persist in a complex ecosystem is

245 influenced by both neutral and adaptive forces (Maignien et al., 2014). Although which of these is the major driver of successful colonization of the human gut remains unclear (Smillie et al., 2018). In the context of FMT, previous studies have suggested neutral processes to determine colonization success based on the abundance of a microbial population in a donor stool sample (Podlesny and Florian Fricke, 2020; Smillie et al.,

250 2018). Indeed, ecological drift may have a significant role in a system dominated by neutral processes, where low-abundance donor populations in the transplant would be less likely to be observed in recipients. In contrast, if the system is dominated by adaptive forces, colonization success would be a function of the population fitness in the recipient environment, rather than its abundance in the transplant.

255 To investigate the impact of neutral versus adaptive processes on colonization in our dataset we first asked whether the prevalence of a donor population in healthy human gut metagenomes, which we define here as a measure of its fitness, was associated with the detection of the same population in donor or recipient metagenomes. Within both FMT cohorts, the mean detection of each population in recipients post-FMT had a stronger

260 association with population fitness than mean detection in donor samples (Figure 2a). The fitness of donor A populations explained $4.2 \%$ of the variation in mean detection of those populations in donor samples $\left(R^{2}=0.042, p=0.021\right)$ and $19 \%$ of variation in mean detection in recipient post-FMT samples $\left(R^{2}=0.19, p=2.7 e-07\right)$, an increase of approximately 4.5-fold (Figure 2a). Similarly, Donor B population fitness explained 7.3\% 
265 of the variation in mean detection in donor samples $\left(R^{2}=0.073, p=2.1 e-04\right)$, and $36 \%$ of the variation in mean detection in recipient post-FMT samples $\left(R^{2}=0.36, p=4.5 e-19\right)$, an increase of approximately 5 -fold (Figure 2a). This suggests that fitness is a better predictor of colonization outcome than it is of the detection of a population in the donor, suggesting that adaptive forces are likely at play. But detecting a donor population in a 270 recipient post-FMT metagenome through metagenomic read recruitment does not prove colonization, since donor genomes can recruit reads from recipient populations that are closely related (i.e., strain variants) and that were low abundance prior to FMT. Singlenucleotide variants in read recruitment results, however, can reveal such cases (Denef, 2019) and quantify their dynamics (Quince et al., 2017). Thus, we developed an improved

275 model that took into consideration the presence and absence of distinct subpopulations in our data and their origins (Supplementary Figure 4). We then used this model to test if colonization success was correlated with population fitness or population dose, which we define here as the relative abundance of a given population in the transplanted donor stool sample. For Donor A populations, colonization outcome was significantly correlated 280 with both dose (Wald test, $A \cup C=0.73, p=7.7 e-05$ ) and fitness (Wald test, $A \cup C=0.76$, $\mathrm{p}=6.3 \mathrm{e}-06$ ) (Figure $2 \mathrm{~b}, \mathrm{c}$ ). But combining both measures as predictive variables did not substantially improve the performance of our colonization model ( $A \cup C=0.82$ ) (Figure 2c). This was likely due to the small, but significant, correlation between dose and fitness in Donor A MAGs $\left(R^{2}=0.053, p=0.0070\right)$ (Figure $2 d$ ). When the fitness of a microbial 285 population is reflected in its relative abundance, the effect of fitness on colonization outcome may be masked by an apparent dose effect. In contrast to Donor $A$, the fitness of Donor B populations and their relative abundance in Donor B samples were not correlated $\left(R^{2}=0.0012, p=0.61\right)$ (Figure $2 d$ ), providing us with an ideal case to analyze these two factors independently. Indeed, there was no correlation between dose of a 290 microbial population in Donor B transplant samples and colonization outcome in recipients post-FMT (Wald test, $A \cup C=0.56, p=0.09$ ). Instead, we found a significant correlation between the fitness of each population and the colonization outcome (Wald test, $A \cup C=0.70, p=9.0 e-07$ ) (Figure 2c).

Taken together, our findings suggest that fitness of a microbial population as measured 295 by its prevalence across global gut metagenomes can predict its colonization success 
better than its abundance in the donor stool sample, giving credence to the role of adaptive rather than neutral ecological processes in colonization. This finding contrasts with previous studies which suggested that the abundance of a given population in the donor sample was an important determinant of colonization (Podlesny and Florian Fricke,

300 2020; Smillie et al., 2018). However, these analyses included many recipient samples collected less than one week after FMT and it is likely that their observations were influenced by the presence of transient populations. Indeed, samples collected immediately after FMT are more likely to inflate the number of colonization events, whereas longitudinal sampling over a longer time course can distinguish transient

305 populations from those that successfully colonized the recipients. We cannot definitively test this hypothesis as we sampled most of our recipients a week after FMT. Still, on average $12 \%$ of the donor populations detected in our recipients a week after FMT were no longer detected after a month (Figure 1, Supplementary Table 3). Overall, our stringent criteria to determine colonization outcome and the extended post-FMT sampling period

310 likely enabled us to study the long-term engraftment of successful and potentially lowabundance colonizers, instead of high-abundance transient populations that may be dominant directly after FMT. 
Donor A
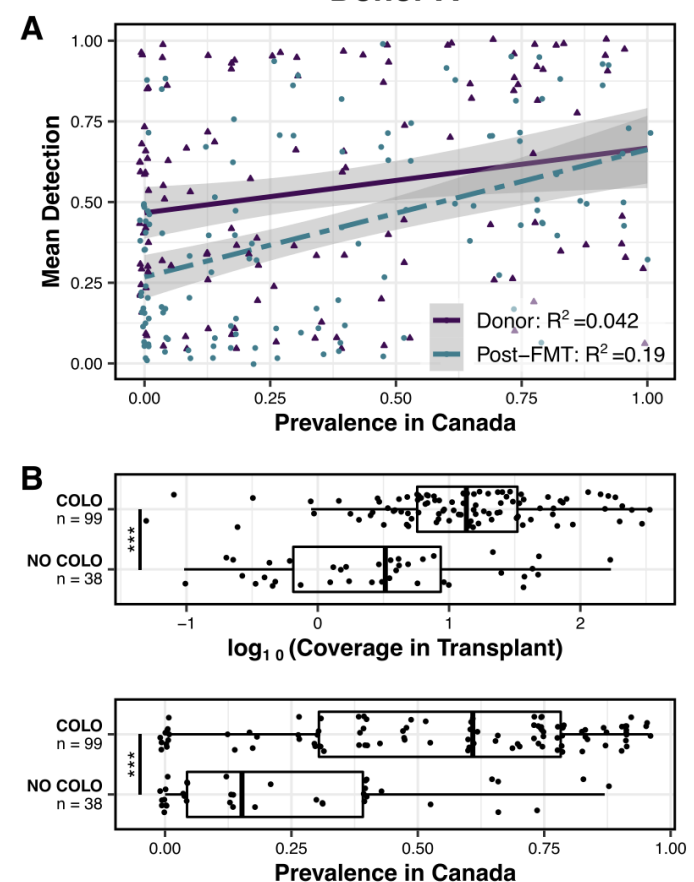

C

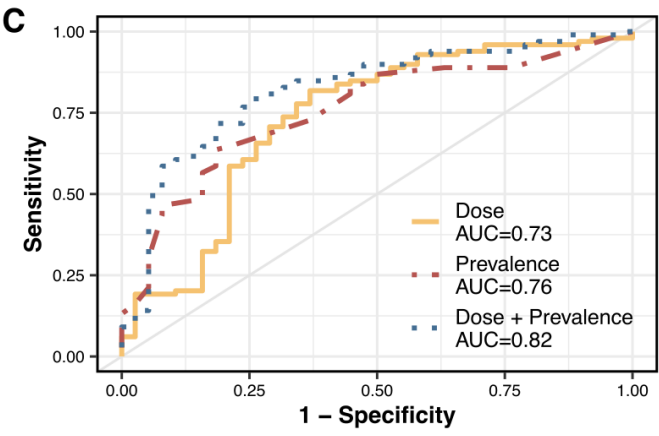

D

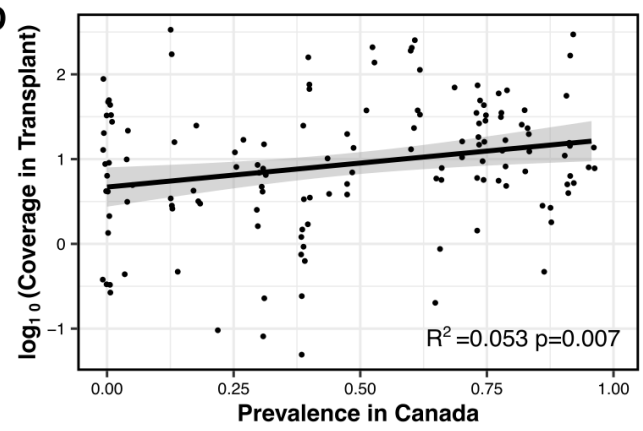

Donor B
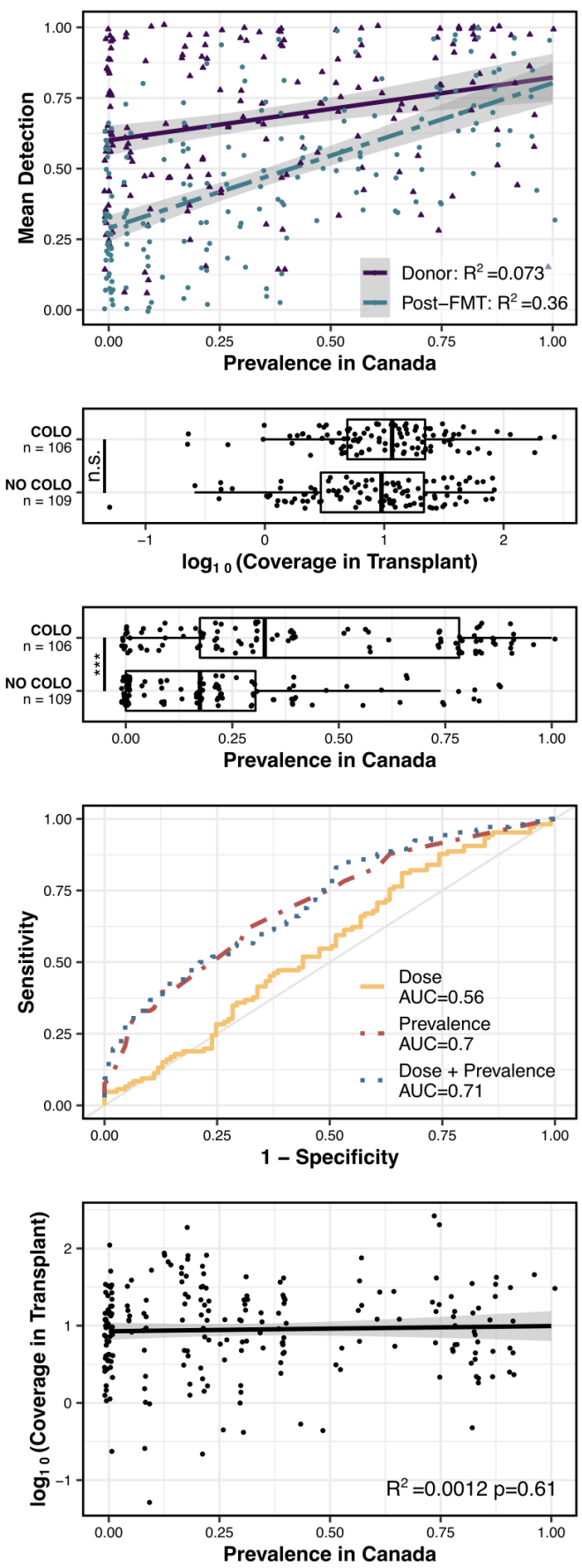

Figure 2. Relationships between dose, prevalence, and colonization outcome. Left: Donor A. Right: Donor B. a) Linear regression models of mean detection of each MAG in either donor or recipient post-FMT samples as a function of prevalence. b) Colonization outcome of MAG/recipient pairs as a function of MAG dose or MAG prevalence. Significance calculated by Wald test. c) Receiver operator curves (ROCs) for logistic regression models of colonization. d) Linear regression models of dose as a function of prevalence. 
Accurately distinguishing the role of dose versus fitness in colonization success is further compounded by the fact that microbial populations that are prevalent across human populations may also tend to be more abundant. This is well illustrated by Donor A. Fortunately, the abundant populations in Donor B did not reflect prevalent microbes in healthy adult guts, which demonstrated the importance of fitness as a determinant of colonization success compared to dose without the confounding effect of a correlation

325 between fitness and dose. Thus, it is a theoretical possibility that colonization success is purely driven by adaptive forces and is not influenced by dose, at all. However, while our data assign a larger role to adaptive forces with confidence, a more accurate determination of the proportional influence of adaptive versus neutral processes in colonization requires a much larger dataset.

330 Colonizer and resilient microbes are enriched in metabolic pathways for the biosynthesis of essential organic compounds

Fitness in a specific environment is conferred to an organism by a combination of functional traits. In the human gut, such traits drive microbial community succession and structure as a response to changing host diet and lifestyle (Koenig et al., 2011; Rothschild

335 et al., 2018). Behind successful colonization and resilience after perturbation are likely similar functional traits that promote fitness. Building on our observation that suggests a primary role of adaptive ecological processes in colonization outcome, we next sought to identify genetic determinants of colonization. For this, we leveraged our access to donor microbial population genomes and global metagenomes to investigate whether a

340 functional enrichment analysis could reveal predictors of success independent of taxonomy.

To generate metabolic insights into colonization success we divided our donor populations into 'high-fitness' and 'low-fitness' groups by considering both their prevalence in FMT recipients and prevalence across global gut metagenomes (Materials

345 and Methods). The 'high-fitness' group included the microbial populations that colonized or persisted in all FMT recipients and were the most prevalent in gut metagenomes from Canada. We assumed that they represented a set of highly fit microbial populations as 
(1) they were able to colonize human gut environments systematically, (2) they persisted in these environments long-term regardless of the host genetics or lifestyle, and (3) they 350 were prevalent in gut metagenomes outside of our study. In comparison, the 'low-fitness' group comprised microbial populations that failed to colonize or persist in at least three FMT recipients. These populations were nevertheless viable gut microbes as not only our long-term sampling of the donors systematically identified them but also, they sporadically colonized some FMT recipients. Yet, unlike those in the high-fitness group, the distribution

355 patterns of low-fitness populations were sparse, not only within our cohort, but also within publicly available metagenomes. In fact, low-fitness populations were less prevalent than high-fitness genomes in each of the 17 different countries we queried, and in countries including United States, Canada, Austria, China, England, and Australia, we detected high-fitness populations in 5 times more people than low-fitness genomes in the same country (Figure 1, Supplementary Table 3). Overall, we conservatively categorized 20 populations in each group for downstream analyses (Supplementary Table 7). All populations in the low-fitness group resolved to Firmicutes. The high-fitness group was also dominated by Firmicutes (15 of 20) but it also included four Bacteroidetes and one Actinobacteria (Supplementary Table 7). Genome completion estimates did not differ 365 between high and low-fitness groups (Wilcoxon rank sum test, $p=0.42$ ) and averaged to $91 \%$ and $93 \%$, respectively. However, genome sizes between the two groups differed dramatically ( $p=2.9 e-06)$, where high-fitness group genomes averaged to $2.8 \mathrm{Mbp}$ while low-fitness group genomes averaged to $1.6 \mathrm{Mbp}$. These results suggest that the length difference between genomes in high and low-fitness groups is likely to have biological

370 relevance. Indeed, we found a very high correspondence between the lengths of our MAGs and their best matching reference genomes in the GTDB $(r=0.88, p=5 e-14)$ (Supplementary Table 7).

Our metabolic enrichment analysis revealed 33 KEGG pathway modules, each containing genes that form a functional unit in a metabolic pathway. Every module that was enriched 375 differentially between these two groups were enriched in the high-fitness group. The lack of any enriched modules in the low-fitness group is in line with the reduction in genome lengths in the low-fitness group and further suggests that the reduction is associated with the absence of metabolic modules. Of all enriched modules, $79 \%$ were modules related 
to biosynthesis, which indicates an overrepresentation of biosynthetic capabilities in the 380 high-fitness group as KEGG modules for biosynthesis only make up 55\% of all KEGG modules (Figure 3, Supplementary Table 7). Of the 33 enriched modules, 48.5\% were associated with amino acid metabolism, 21.2\% with vitamin and cofactor metabolism, $18.2 \%$ with carbohydrate metabolism, $6 \%$ with lipid metabolism and 3\% with energy metabolism (Supplementary Table 7). Metabolic modules that were enriched in the high-

385 fitness group included the biosynthesis of seven of the nine essential amino acids, indicating the importance of metabolic competency to synthesize high-demand compounds as a factor increasing fitness in colonizing new gut environments (Supplementary Table 7). This is further supported by the enrichment of biosynthesis pathways for the essential cofactor vitamin B12 (cobalamin), which occurred in $67.5 \%$ of

390 the high-fitness populations and only $12.5 \%$ of the low-fitness group (Supplementary Table 7). Vitamin B12 is structurally highly complex and costly to produce, requiring expression of more than 30 genes that are exclusively encoded by bacteria and archaea (Martens et al., 2002). Thus, the competitive advantages conferred by metabolic autonomy appear to outweigh the additional costs. In addition to the biosynthesis of 395 tetrahydrofolate, riboflavin, and cobalamin, the high-fitness group had a larger representation of biosynthetic modules for vitamins including biotin, pantothenate, folate, and thiamine (Supplementary Table 7), micronutrients that are equally important in bacterial and human metabolism and are shown to play important roles in mediating hostmicrobe interactions (Biesalski, 2016). Interestingly, enriched metabolic modules in our 400 analysis partially overlap with those that Feng et al. identified as the determinants of microbial fitness using metatranscriptomics and a germ-free mouse model conventionalized with microbial isolates of human origin (Feng et al., 2020).

Even though enriched metabolic modules occurred mostly in high-fitness populations, we did find some of these modules in the low-fitness group as well (Supplementary Table 7), 405 but their distribution was not uniform as they primarily occurred only in a subset of genomes that resolved to Firmicutes (Figure 3). We then sought to identify whether the levels of completion of these modules that occurred in both groups were identical. For this, we matched six low-fitness genomes that encoded modules enriched in high-fitness group genomes to six high-fitness genomes from the same phylum (marked as HF and 
410 LF subgroups in Figure 3). Bacterial single-copy core genes estimated that genomes in both subgroups were highly complete with a slight increase in average completion of lowfitness genomes (93.7\%) compared to high-fitness genomes (90.1\%). Despite the higher estimated genome completion for low-fitness populations, estimated metabolic module completion values were significantly lower in the low-fitness group (Wilcoxon rank sum

415 test with continuity correction, $V=958, p=5 e-09$ ) (Figure 3, Supplementary Table 7). This indicates that even when modules that are associated with high-fitness were detected in low-fitness genomes, they were systematically missing genes and were less complete than the same modules in high-fitness genomes.

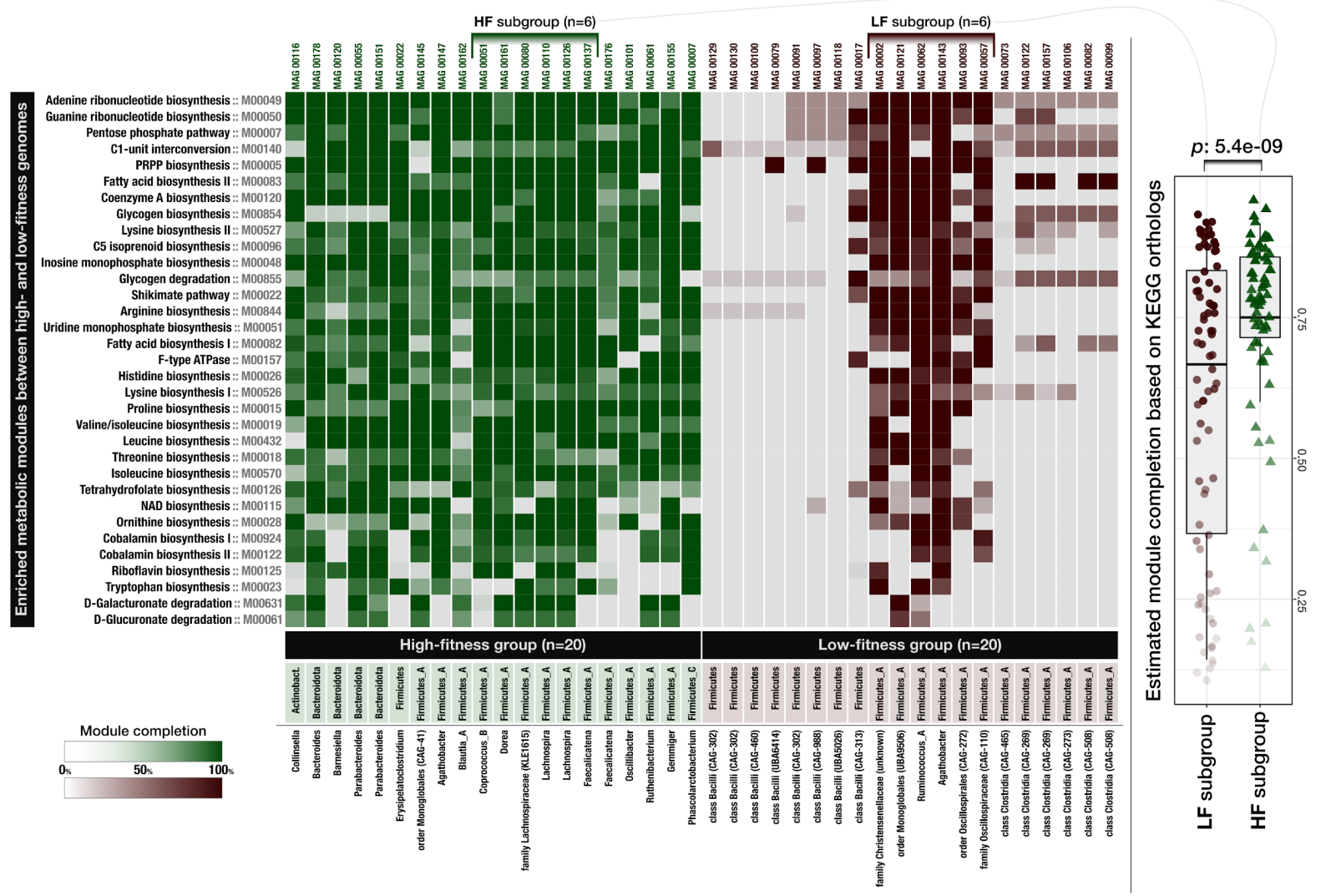

420 Figure 3. Distribution of metabolic modules across low and high-fitness genomes. Each data point in this heat map shows the level of completion of a given metabolic module (rows) in a given genome (columns). The box-plot on the right-side compares a subset of low-fitness (LF) and high-fitness (HF) genomes, where each data point is the level of completion of a given metabolic module in a genome and shows a statistically significant difference between the overall completion of metabolic modules between these subgroups 425 (Wilcoxon rank sum test, $\mathrm{p}=5.4 \mathrm{e}-09$ ). 


\section{While gut microbial ecosystems of healthy individuals include both low- and high-fitness microbes, IBD primarily selects for high- fitness populations}

Our results so far show that while the healthy donor environment could support both high430 fitness and low-fitness populations (Figure 1, Supplementary Table 3), challenging microbes to colonize a new environment or to withstand massive ecosystem perturbation during FMT selects for high-fitness populations (Figure 3, Supplementary Table 7), suggesting that metabolic competence is a more critical determinant of fitness during stress than during homeostasis. Based on these observations, it is conceivable to

435 hypothesize that (1) a gut environment in homeostasis will support a range of microbial populations with a wide spectrum of metabolic competency, and (2) a gut environment under stress will select for high metabolic competency in microbial populations.

To test these hypotheses, we compared genomes reconstructed from a cohort of healthy individuals (Pasolli et al., 2019) to genomes reconstructed from individuals who were

440 diagnosed with inflammatory bowel disease (IBD). Our IBD dataset was composed of two cohorts: a set of patients with pouchitis (Vineis et al., 2016), a form of IBD with similar pathology to ulcerative colitis (De Preter et al., 2009), and a set of pediatric Crohn's disease patients (Quince et al., 2015). The number of genomes per individual and the average level of genome completeness per group were similar between healthy

445 individuals and those with IBD: overall, our analysis compared 264 genomes from 22 healthy individuals with an average completion of $90.4 \%, 44$ genomes from 4 pouchitis patients with an average completion of $89.2 \%$ and 256 genomes from 12 Crohn's disease patients with an average completion of $94.1 \%$ (Supplementary Table 8). Intriguingly, similar to the length differences between genomes of high-fitness and low-fitness 450 populations (2.8 Mbp versus 1.6 Mbp on average), microbial populations associated with IBD patients had larger genomes compared to healthy people and averaged to $3.0 \mathrm{Mbp}$ versus 2.6 Mbp, respectively (Supplementary Table 8). This suggests that despite the comparable levels of completion of microbial genomes from the healthy cohort, these 
genomes tended to be smaller in size compared to those reconstructed from individuals 455 with IBD.

Next, we asked whether the completion of those metabolic modules associated with colonization success and resilience differed between the genomes reconstructed from healthy and IBD individuals. The level of completion of the 33 metabolic modules were almost identical between high-fitness genomes and genomes from IBD patients

460 (Wilcoxon rank sum test, $\mathrm{p}=0.5$ ), but genomes from healthy individuals were significantly less complete compared to high-fitness genomes (Wilcoxon rank sum test, $p<1 e-07$ ) as well as genomes from IBD patients (Wilcoxon rank sum test, $p<1 e-07$ ) (Figure 4, Supplementary Table 8). Metabolic modules with the largest differences in completion between genomes from healthy and IBD individuals included biosynthesis of cobalamin, 465 arginine, ornithine, tryptophan, isoleucine as well as the Shikimate pathway (Figure 4, Supplementary Table 8), a seven step metabolic route bacteria use for the biosynthesis of aromatic amino acids (phenylalanine, tyrosine, and tryptophan) (Herrmann and Weaver, 1999).

Our findings show that the same set of key metabolic modules that distinguish high-fitness 470 and low-fitness populations in FMT were also differentially associated with populations that occurred in healthy individuals compared to IBD patients. In particular, while healthy individuals seem to harbor microbes with a broad range of metabolic competency, individuals who suffer from two different forms of IBD appear to harbor organisms with higher metabolic autonomy. It is conceivable that a stable gut microbial ecosystem is 475 more likely to support low-fitness populations through metabolic cross-feeding, where vitamins, amino acids, and nucleotides are exchanged between microbes (D'Souza et al., 2018). In contrast, host-mediated environmental stress in IBD likely disrupts such interactions and creates an ecological filter that selects for metabolic competence, which subsequently leads to loss of diversity and the dominance of organisms with large 480 genomes that are not necessarily abundant in states of homeostasis. 


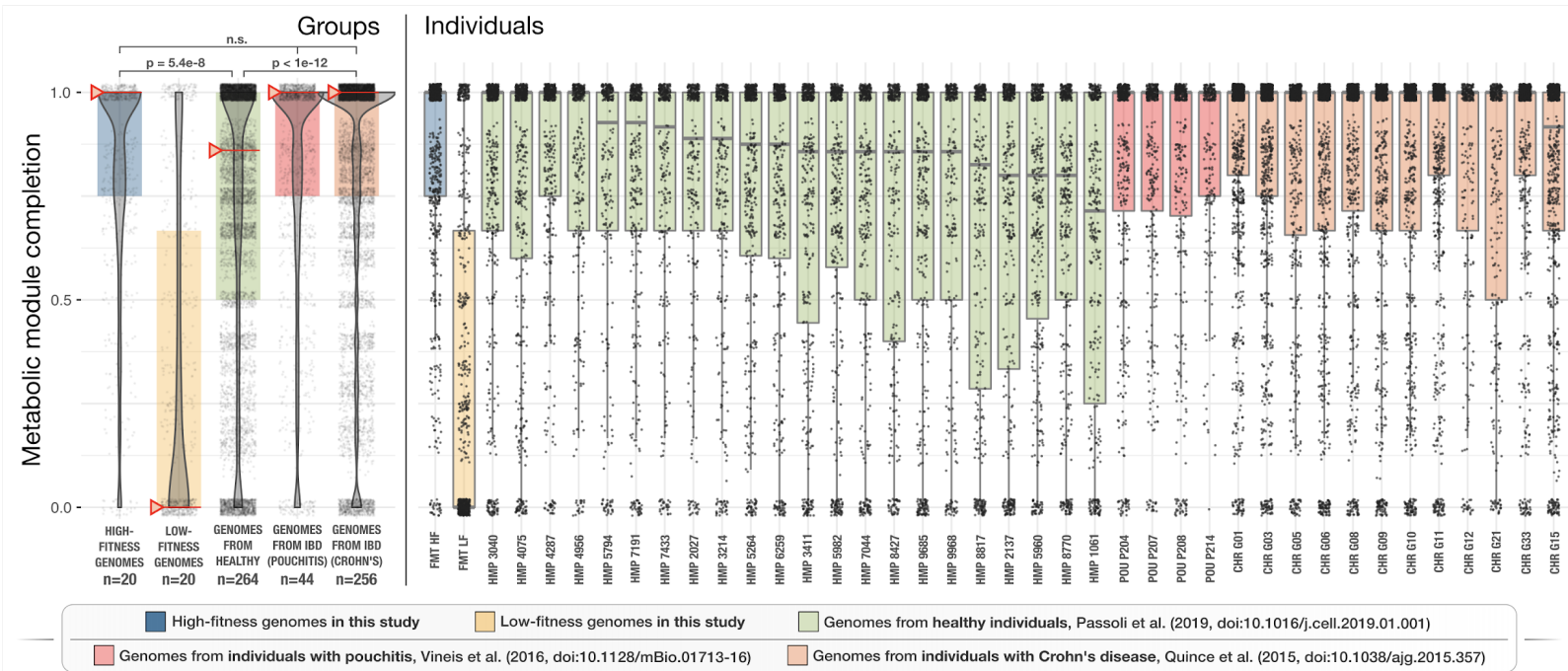

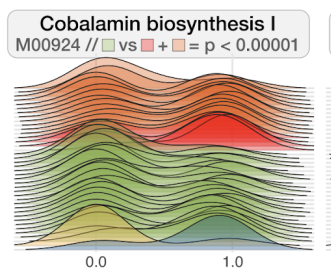

Fatty acid biosynthesis I M00082// $\square \mathrm{vs} \square+\square=\mathrm{p}<0.00001$

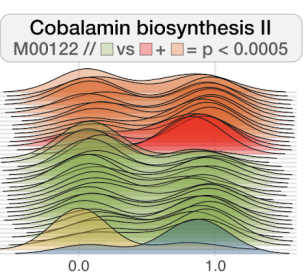

Arginine biosynthesis

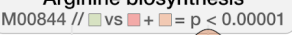

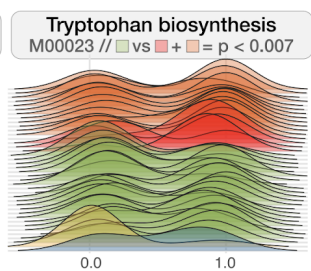

Proline biosynthesis M00015// $\square$ vs $\square++=p<0.00001$

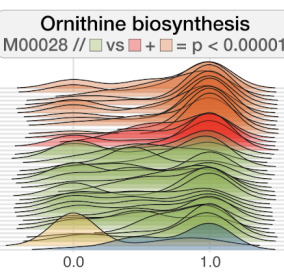

Lysine biosynthesis
Isoleucine biosynthesis
M00570// $\square$ vs $\square+\square=\mathrm{p}<0.0000$

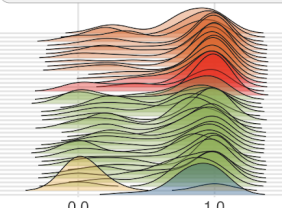

Shikimate pathway Shikimate pathway
M00022 // $\square$ vs $\square+\square=p<0.00001$
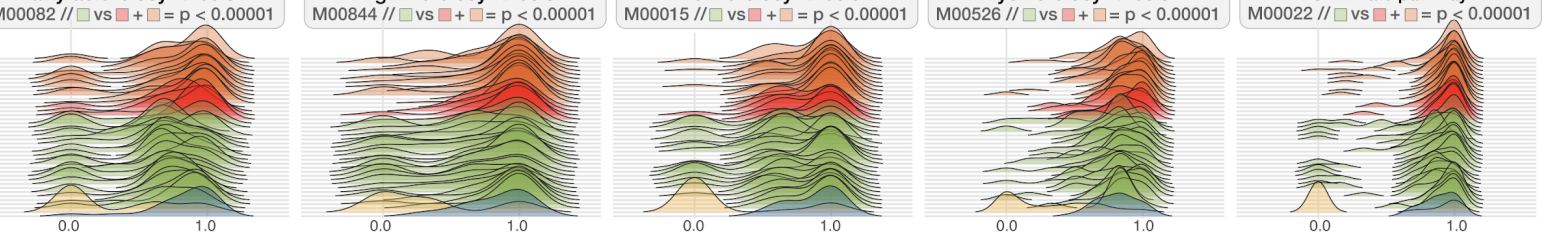

Figure 4. Distribution of metabolic modules in genomes reconstructed from healthy individuals and individuals with IBD. The top panel shows the metabolic module completion values for (1) high- and (2) lowfitness donor genomes identified in this study (blue and yellow), (3) genomes from healthy individuals (green), and (4) genomes from individuals with pouchitis (red) and Crohn's disease (orange). Red whiskers in group averages indicate the median. Next to group averages shown the distribution of metabolic modules for each individual. Each dot in a given box-plot represents one of 33 metabolic modules that were enriched in high-fitness FMT donor populations and the y-axis indicates its estimated completion. In the bottom panel the completion values for 10 of the 33 pathways demonstrated as ridge-line plots. Each plot represents a single metabolic module where each layer corresponds to an individual, and the shape of the layer represents the completion of a given metabolic module across all genomes reconstructed from that individual.

These observations have implications on our understanding of the hallmarks of healthy gut environments from an ecological point of view. Defining the 'healthy gut microbiome' 495 has been a major goal of human gut microbiome research (Bäckhed et al., 2012), and remains elusive (Eisenstein, 2020). Despite comprehensive investigations that 
considered core microbial taxa (Arumugam et al., 2011; Lloyd-Price et al., 2016) or guilds of microbes that represent coherent functional groups (Wu et al., 2021), the search for 'biomarkers' of healthy microbiomes is ongoing (McBurney et al., 2019). Given our data

500 we hypothesize that one of the defining features of a healthy gut environment is its ability to support a diverse community of microbes with a broad spectrum of metabolic competence, where both low-fitness and high-fitness populations live in a coherent ecosystem. Conversely, an enrichment of metabolically competent high-fitness populations would likely indicate the presence of environmental stress. Our analyses

505 demonstrate that this is a quantifiable feature of microbial communities through genomeresolved metagenomic surveys. Our analyses have limitations. For instance, metabolic insights in our study have been limited to genomic potential and have considered only well-known metabolic pathways, which, given the extent of the unknown coding space in microbial genomes (Vanni et al., 2020), are likely far from complete. As a result, the

510 disproportional enrichment of biosynthetic modules in high-fitness genomes indicates that the ability to synthesize essential biological compounds is necessary but likely insufficient to survive environmental stress in the gut. Nevertheless, the finding that the same metabolic modules that promote colonization success after FMT are also the hallmarks of fitness in IBD suggests the presence of ecological principles that are shared between

515 these systems and warrants deeper investigation.

\section{Subtle differences in key functions distinguish populations of the same genus with differential colonization success}

While adaptive processes that favor metabolic independence explain the determinants of colonization and resilience for distantly related taxa, metabolic features that promote high-

520 fitness at this broad level may not explain differences in fitness between more closely related taxa, such as distinct species within a single genus, which are likely to have similar metabolic capabilities (Martiny et al., 2013) due to unifying ecological traits in higher ranks of taxonomy (Philippot et al., 2010). We finally investigated whether we could identify determinants of fitness across metabolically similar populations with different levels of

525 success in colonizing unrelated individuals. 
Members of the genus Bifidobacterium have long been used as probiotics (Gomes and Malcata, 1999) and are prevalent occupants of the healthy human gut microbiota (Arboleya et al., 2016). In our dataset, Bifidobacterium was the second most abundant genus (14.1\%) after Bacteroides (15.8\%) in Donor A, from whom we reconstructed three

530 MAGs over $98 \%$ completion that resolved to three distinct species in this genus: $B$. longum (DA_MAG_00052), B. adolescentis subsp. adolescentis (DA_MAG_00018), and B. animalis subsp. lactis (DA_MAG_00011, Supplementary Table 3). While each of these Bifidobacterium populations occurred in Donor A metagenomes in a relatively stable fashion, they showed vastly different colonization efficiency upon FMT (Figure 5),

535 enabling us to investigate determinants of colonization among closely related taxa.

In contrast to the $B$. longum and $B$. adolescentis subsp. adolescentis (henceforth $B$. adolescentis) populations that colonized most recipients, B. animalis subsp. lactis (henceforth $B$. lactis) did not seem to have colonized any of our recipients (Supplementary Table 3). Overall, we were able to detect B. Iongum, B. adolescentis, and B. lactis 540 populations in $83 \%, 75 \%$, and $4 \%$ of all post-FMT recipient metagenomes, respectively (Figure 5). Most strikingly, patterns of colonization that emerged from the analysis of FMT recipients reflected those seen in publicly available gut metagenomes from Canada, where $B$. longum, B. adolescentis, and B. lactis populations occurred in $74 \%, 39 \%$, and $13 \%$ of the population, demonstrating a positive relationship (Pearson's correlation of 0.9 ,

545 n.s.) between the colonization efficiency upon FMT and the fitness of these populations. Furthermore, the gut metagenomes from 17 countries confirmed the substantially reduced fitness of $B$. lactis globally (Supplementary Table 9). Interestingly, the $B$. lactis MAG we reconstructed from Donor A was virtually identical (with over $99.99 \%$ sequence identity over $99.82 \%$ alignment, Supplementary Table 9 ) to most $B$. lactis strains that are 550 widely used as probiotics (Jungersen et al., 2014), revealing a disagreement between the preferences of commercial microbial therapeutics and human gut microbial ecology. 

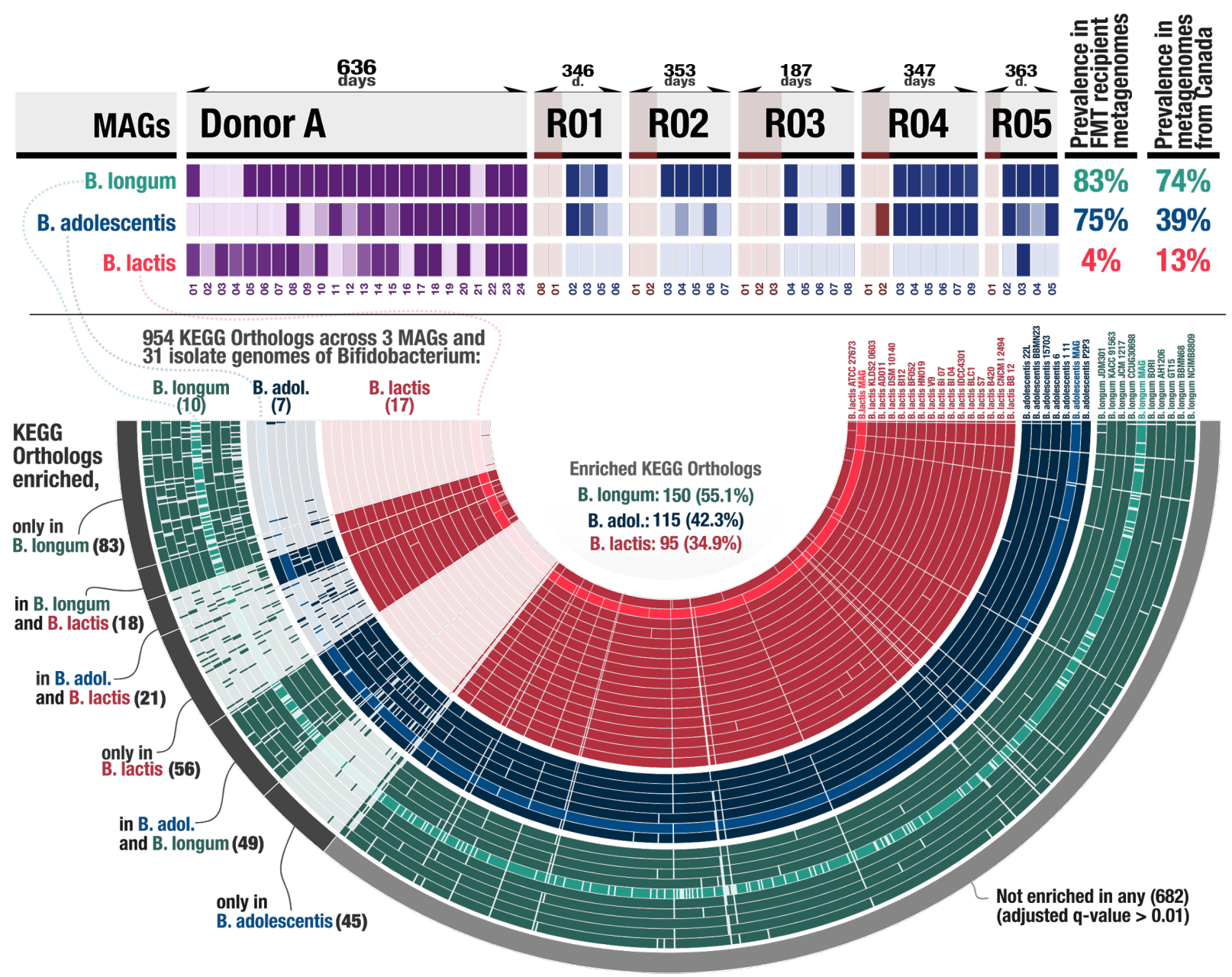

Figure 5. Characteristics of three Bifidobacterium species. Top panel shows the distribution of Donor A MAGs that represent three distinct Bifidobacterium populations across donor and recipient metagenomes before and after FMT.

The last two columns in this panel show the prevalence of these populations in post-FMT metagenomes, and publicly available gut metagenomes from Canada. The panel below displays the distribution of KEGG orthologs across the three Bifidobacterium MAGs along with 31 high-quality isolate genomes from the NCBI. Each item shown in concentric circles represents a single function assigned by the database of KEGG Orthologs, and each layer is a distinct genome. The intensity of color indicates the presence of a given function in a given genome. The most outer circle marks groups 560 of functions that are enriched in various groups of Bifidobacterium genomes as well as those functions that are not enriched in any group as they are either in all genomes, or only a very small number of them.

To identify factors that may explain the differences in colonization success between $B$. longum, B. adolescentis, and B. lactis, we created a collection of Bifidobacterium genomes that, in addition to the three metagenome-assembled genomes we 565 reconstructed, included 31 complete genomes obtained from the NCBI (within-group and across-groups average gANI of $98.9 \%$ and $77.3 \%$, respectively) (Supplementary Table 
9). All three groups of Bifidobacterium genomes encoded the majority of the metabolic pathways associated with the high-fitness group (63\% $\pm 5 \%)$. However, missing pathways were not uniformly distributed across three: $B$. lactis lacked the largest fraction of these

570 pathways (42\%) compared to the more prevalent $B$. adolescentis $(36 \%)$ and $B$. longum (33\%) (Supplementary Table 9). B. Iongum and B. adolescentis carried the complete tetrahydrofolate (vitamin B9) biosynthesis pathway in agreement with previous metabolic descriptions of Bifidobacterium (D'Aimmo et al., 2012; Sugahara et al., 2015) which qualifies this group as attractive probiotics (Pompei et al., 2007; Strozzi and Mogna,

575 2008), but this pathway was absent in $B$. lactis genomes. We also found that $B$. longum and $B$. adolescentis genomes encoded histidine biosynthesis which $B$. lactis lacked (Supplementary Table 9). Finally, the average genome lengths of $B$. longum (2.31 Mbp) and $B$. adolescentis $(2.18 \mathrm{Mbp})$ were longer than the average genome length of $B$. lactis (1.94 Mbp), which reflects the pattern we observed previously where high-fitness

580 populations tended to have larger genomes. In summary, even though all Bifidobacterium genomes in our pangenome had a higher metabolic overlap with one another compared to high-fitness and low-fitness genomes we have previously studied, the reduced fitness of $B$. lactis compared to $B$. longum and $B$. adolescentis could still be explained by the absence of a small number of metabolic competencies associated with the high-fitness

585 group genomes.

Next, we focused on the enrichment of individual functions across the three groups of genomes using gene annotations from KOfam profiles (Aramaki et al., 2020) from the Kyoto Encyclopedia of Genes and Genomes (KEGG) (Kanehisa and Goto, 2000) and Clusters of Orthologous Groups (COGs) from the NCBI (Galperin et al., 2021). Of all 954

590 unique KOfams found in our Bifidobacterium pangenome, 272 functions were not common to all genomes but statistically enriched in either one or two groups. Our analysis of these accessory functions showed that $B$. longum encoded $150(55.5 \%), B$. adolescentis encoded 115 (42.3\%), and B. lactis encoded 95 (34.9\%) of all accessory functions that were statistically enriched (Figure 5, Supplementary Table 9). The same 595 analysis with 1,286 unique COGs confirmed these observations: of all 353 COGs enriched in any group, B. longum encoded 212 (60.1\%), B. adolescentis encoded 172 $(48.7 \%)$, and B. lactis encoded 118 (33.4\%) (Supplementary Table 9). Overall, these 
results reveal a striking correlation between the number of accessory functions associated with B. longum, B. adolescentis, and B. lactis, and echo the absence of 600 metabolic pathways in B. lactis even at the level of accessory gene functions, explaining their differential ability to colonize new individuals and distribution in global human gut metagenomes.

We finally investigated the contents of the differentially occurring accessory functions to speculate on whether they could be related to differences in fitness. For instance, in

605 contrast to all $B$. longum and $B$. adolescentis in the Bifidobacterium pangenome, none of the $B$. lactis genomes encoded a phosphoenolpyruvate phosphotransferase (PEP-PTS) system specific for the uptake of $\beta$-glucoside (Supplementary Table 9). As the genus Bifidobacterium is characterized by a large array of genes associated with carbohydrate uptake and metabolism (Kleerebezem and Vaughan, 2009; Schell et al., 2002; Ventura

610 et al., 2009), B. lactis represents a notable exception with a lower number of genes associated with carbohydrate metabolism, fewer genes encoding carbohydrate-specific ABC transporters, and the absence of phosphoenolpyruvate-phosphotransferase (PEPPTS) systems (Barrangou et al., 2009). The absence of any other PEP-PTS system in $B$. longum and $B$. adolescentis may indicate the catabolic niche occupied by these

615 populations in the human gut that is shaped by their extensive capacity for uptake and metabolism of plant derived glycosides (Chien et al., 2006; Schell et al., 2002). Additional functions that exclusively occurred in $B$. adolescentis and $B$. longum genomes included two multidrug resistance pumps of the 'multidrug and toxin extrusion' (MATE) type, three transporters of the major facilitator superfamily (MFS) involved in bile acid tolerance and 620 macrolide efflux, two bile acid:natrium ion symporters, and one proton/chloride ion antiporter conferring acid tolerance (Supplementary Table 9). The drug defense mechanisms may act to protect these populations during periods of inflammation and drug administration, but may also be beneficial with regard to the common ingestion of antibiotics through various food products (Kirchhelle, 2018). These results show that in 625 the microbial fitness landscape of the human gut, where the determinants of success across distantly related taxa are primarily defined by the presence or absence of a large number of metabolic pathways, there exists smaller niches equally accessible to closely 
related organisms with similar metabolic potential, among which success can be speculated by subtle differences in key functions.

630 Conclusions

Our study points to adaptive ecological processes as primary determinants of both longterm colonization after FMT and microbial fitness in the human gut environment through metabolic competency as conferred by biosynthesis of nucleotides, amino acids, and essential micronutrients. Even when we found these metabolic modules in low-fitness

635 populations, they were systematically less complete compared to their high-fitness counterparts.

Our findings suggest that in a healthy gut environment high- and low-fitness populations co-occur in harmony, with their differential fitness indiscernible by taxonomy or relative abundance. However, transfer to a new gut environment through FMT, or host-mediated

640 stress through IBD, initiates an ecological filter that selects for high-fitness populations that can self-sustain. This model offers a null hypothesis to explain how low-abundance members of healthy gut environments can come to dominate the gut microbiota under stressful conditions, while not being causally associated with disease states. If the association between particular microbial taxa and disease is solely driven by their

645 superior metabolic competence, microbial therapies that aim to treat complex diseases by adding microbes associated with healthy individuals will be unlikely to compete with the adaptive processes that regulate complex gut microbial ecosystems.

\section{Materials and Methods}

Sample collection and storage. We used a subset of individuals who participated in a 650 randomized clinical trial (Kao et al., 2017) and conducted a longitudinal FMT study of two human cohorts (DA and DB), each consisting of one FMT donor and 5 FMT recipients of that donor's stool. All recipients received vancomycin for a minimum of 10 days pre-FMT at a dose of $125 \mathrm{mg}$ four times daily. Three DA and two DB recipients received FMT via 
pill, and two DA and three DB recipients received FMT via colonoscopy. All recipients had 655 recurrent $C$. difficile infection before FMT, and two DA recipients and $1 \mathrm{DB}$ recipient were also diagnosed with ulcerative colitis (UC). 24 stool samples were collected from the DA donor over a period of 636 days, and 15 stool samples were collected from the DB donor over a period of 532 days. Between 5 and 9 stool samples were collected from each recipient over periods of 187 to 404 days, with at least one sample collected pre-FMT and

6602 samples collected post-FMT. This gave us a total of 109 stool samples from all donors and recipients. Samples were stored at $-80^{\circ} \mathrm{C}$. (Supplementary Figure 1, Supplementary Table 1)

Metagenomic short-read sequencing. We extracted the genomic DNA from frozen samples according to the centrifugation protocol outlined in MoBio PowerSoil kit with the

665 following modifications: cell lysis was performed using a GenoGrinder to physically lyse the samples in the MoBio Bead Plates and Solution (5-10 min). After final precipitation, the DNA samples were resuspended in TE buffer and stored at $-20{ }^{\circ} \mathrm{C}$ until further analysis. Sample DNA concentrations were determined by PicoGreen assay. DNA was sheared to $\sim 400$ bp using the Covaris S2 acoustic platform and libraries were constructed

670 using the Nugen Ovation Ultralow kit. The products were visualized on an Agilent Tapestation 4200 and size-selected using BluePippin (Sage Biosciences). The final library pool was quantified with the Kapa Biosystems qPCR protocol and sequenced on the Illumina NextSeq500 in a $2 \times 150$ paired-end sequencing run using dedicated read indexing.

675 'Omics workflows. Whenever applicable, we automated and scaled our 'omics analyses using the bioinformatics workflows implemented by the program 'anvi-run-workflow' (Shaiber et al., 2020) in anvi'o (Eren et al., 2015, 2021). Anvi'o workflows implement numerous steps of bioinformatics tasks including short-read quality filtering, assembly, gene calling, functional annotation, hidden Markov model search, metagenomic read680 recruitment, metagenomic binning, pangenomics, and phylogenomics. Workflows use Snakemake (Köster and Rahmann, 2012) and a tutorial is available at the URL http://merenlab.org/anvio-workflows/. The following sections detail these steps. 
Taxonomic composition of metagenomes based on short reads. We used Kraken2 v2.0.8-beta (Wood et al., 2019) with the NCBl's RefSeq bacterial, archaeal, viral and viral

685 neighbours genome databases to calculate the taxonomic composition within short-read metagenomes.

Assembly of metagenomic short reads. To minimize the impact of random sequencing errors in our downstream analyses, we used the program 'iu-filter-quality-minoche` to process short metagenomic reads, which is implemented in illumina-utils v2.11 (Eren et

690 al., 2013) and removes low-quality reads according to the criteria outlined by Minoche et al. (Minoche et al., 2011). IDBA_UD v1.1.2 (Peng et al., 2012) assembled quality-filtered short reads into longer contiguous sequences (contigs), although we needed to recompile IDBA_UD with a modified header file so it could process $150 \mathrm{bp}$ paired-end reads.

Processing of contigs. We use the following strategies to process both sequences we 695 obtained from our assemblies and those we obtained from reference genomes. Briefly, we used (1) 'anvi-gen-contigs-database` on contigs to compute k-mer frequencies and identify open reading frames (ORFs) using Prodigal v2.6.3 (Hyatt et al., 2010), (2) `anvirun-hmms` to identify sets of bacterial (Campbell et al., 2013) and archaeal (Rinke et al., 2013) single-copy core genes using HMMER v3.2.1 (Eddy, 2011), (3) `anvi-run-ncbi-

700 cogs 'to annotate ORFs with functions from the NCBI's Clusters of Orthologous Groups (COGs) (Tatusov et al., 2003), and (4) 'anvi-run-kegg-kofams' to annotate ORFs with functions from the KOfam HMM database of KEGG orthologs (KOs) (Aramaki et al., 2020; Kanehisa and Goto, 2000). To predict the approximate number of genomes in metagenomic assemblies we used the program 'anvi-display-contigs-stats', which

705 calculates the mode of the frequency of single-copy core genes as described previously (Delmont and Eren, 2016).

Metagenomic read recruitment, reconstructing genomes from metagenomes, determination of genome taxonomy and ANI. We recruited metagenomic short reads to contigs using Bowtie2 v2.3.5 (Langmead and Salzberg, 2012) and converted resulting

710 SAM files to BAM files using samtools v1.9 (Li et al., 2009). We profiled the resulting BAM files using the program 'anvi-profile' with the flag '--min-contig-length` set to 2500 to eliminate shorter sequences to minimize noise. Once we have read recruitment results 
from each metagenome is profiled to store contig coverages into single anvi'o profile databases, 'anvi-merge' combined all profiles into an anvi'o merged profile for 715 downstream visualization, binning, and statistical analyses. We then used 'anvi-clustercontigs' to group contigs into 100 initial bins using CONCOCT v1.1.0 (Alneberg et al., 2014), 'anvi-refine' to manually curate initial bins with conflation error based on tetranucleotide frequency and differential coverage signal across all samples, and 'anvisummarize to report final summary statistics for each gene, contig, and bin. We used the 720 program 'anvi-rename-bins' to identify bins that were more than $70 \%$ complete and less than $10 \%$ redundant, and store them in a new collection as metagenome-assembled genomes (MAG), discarding lower quality bins from downstream analyses. GTBD-tk v0.3.2 (Chaumeil et al., 2019) assigned taxonomy to each of our MAG using GTDB r89 (Parks et al., 2018), but to assign species- and subspecies-level taxonomy for

725 'DA_MAG_00057', 'DA_MAG_00011', 'DA_MAG_00052' and 'DA_MAG_00018', we used 'anvi-get-sequences-for-hmm-hits' to recover DNA sequences for bacterial singlecopy core genes that encode ribosomal proteins, and searched them in the NCBI's nucleotide collection (nt) database using BLAST (Altschul et al., 1990). Finally, the program 'anvi-compute-genome-similarity' calculated pairwise genomic average

730 nucleotide identity (gANI) of our genomes using PyANI v0.2.9 (Pritchard et al., 2016).

Criteria for MAG detection in metagenomes. Using mean coverage to assess the occurrence of populations in a given sample based on metagenomic read recruitment can yield misleading insights since this strategy cannot accurately distinguish reference sequences that represent very low-abundance environmental populations from those

735 sequences that do not represent an environmental population in a sample yet still recruit reads from non-target populations due to the presence of conserved genomic regions. Thus, we relied upon the 'detection' metric, which is a measure of the proportion of the nucleotides in a given sequence that are covered by at least one short read, and considered a population was detected in a metagenome if anvi'o reported a detection

740 value of at least 0.25 for its genome (whether it was a metagenome-assembled or isolate genome). Values of detection in metagenomic read recruitment results often follow a bimodal distribution for populations that are present and absent (see Supplementary 
Figure 2 in (Utter et al., 2020)), thus 0.25 is an appropriate cutoff to eliminate false-positive signal in read recruitment results for populations that are absent.

745 Identification of MAGs that represent multiple subpopulations. To identify subpopulations of MAGs in metagenomes, we used the anvi'o command 'anvi-genvariability-profile` with the '--quince-mode` flag which exported single-nucleotide variant (SNV) information for all MAGs after read recruitment. We then used DESMAN v2.1.1 (Quince et al., 2017) to analyze SNVs to determine the number and distribution of

750 subpopulations represented by a single genome. To account for non-specific mapping that can inflate the number of estimated subpopulations, we removed any subpopulation that made up less than $1 \%$ of the entire population explained by a single MAG. To account for noise due to low-coverage, we only investigated subpopulation for MAGs for which the mean non-outlier coverage of single-copy core genes was at least 10X.

755 Criteria for colonization of a recipient by a MAG. We developed a method to determine whether or not a MAG successfully colonized a recipient, and applied this method to each MAG and each recipient within a cohort. In order to confidently assign colonization or non-colonization phenotypes to each MAG/recipient pair, we required that the MAG be detected in the donor sample used for transplant into the recipient. If these criteria were 760 met, we then determined whether the MAG was detected in any post-FMT recipient sample taken more than 7 days after transplant. If not, the MAG/recipient pair was considered a non-colonization event. If the MAG was detected in the recipient greater than7 days post-FMT, we used subpopulation information to determine if any subpopulation present in the donor and absent in the recipient pre-FMT was detected in

765 the recipient more than 7 days post-FMT. If this was the case, we considered this to represent a colonization event. See Supplementary Figure 4 for a complete outline of all possible cases.

Determination of dose and fitness for MAGs. We defined population dose as the second and third quartile mean coverage of a population in the transplanted stool sample.

770 We defined fitness as the prevalence of a population in 23 healthy adult gut metagenomes (see Materials and Methods: Criteria for MAG detection in metagenomes) from Canada, the same country in which the FMTs were performed. 
Regression analysis. To examine the association between dose and/or prevalence with colonization outcome, we built binomial logistic regression models using the R stats ' $\mathrm{glm}$ '

775 function. We used the R stats 'predict' function and the R pROC 'roc' function to evaluate our models by creating receiver operating characteristic (ROC) curves and calculating the area under the ROC curve (AUC). To determine the correlation between dose and prevalence, we performed linear regression using the R stats 'Im' function. We used the $\mathrm{R}$ tidyverse package, including ggplot2, to visualize boxplots, scatterplots, and ROC 780 curves.

Pangenomic analysis and gANI. We used anvi'o to compute and visualize pangenomes of MAGs and reference genomes. We stored all processed MAG and reference genome contigs (see Contig processing methods section) in an anvi'o database using the command 'anvi-gen-genomes-storage'. To create the pangenomes, we then passed that

785 database to the command 'anvi-pan-genome` which used NCBI's BLAST (Altschul et al., 1990) to quantify gene similarity within and between genomes and the Markov Cluster algorithm (MCL) (Enright et al., 2002) to cluster groups of similar genes. We set the 'anvipan-genome '--min-occurrence' flag to 2 to remove gene clusters only present in one genome (singletons), and visualized pangenomes using `anvi-display-pan`.

790 Phylogenomic tree construction. To concatenate and align amino acid sequences of 46 single-copy core (Campbell et al., 2013) ribosomal proteins that were present in all of our Bifidobacterium MAGs and reference genomes, we ran the anvi'o command 'anviget-sequences-for-hmm-hits' with the '--return-best-hit', '--get-aa-sequence' and '-concatenate` flags, and the '--align-with` flag set to 'muscle' to use MUSCLE v3.8.1551

795 (Edgar, 2004) for alignment. We then ran 'anvi-gen-phylogenomic-tree' with default parameters to compute a phylogenomic tree using FastTree 2.1 (Price et al., 2010).

Analysis of metabolic modules and enrichment. We calculated the level of completeness for a given KEGG module (Kanehisa et al., 2014, 2017) in our genomes using the program 'anvi-estimate-metabolism', which leveraged previous annotation of 800 genes with KEGG orthologs (KOs) (see the section 'Processing of contigs'). Then, the program 'anvi-compute-functional-enrichment' determined whether a given metabolic module was enriched in based on the output from 'anvi-estimate-metabolism”. The URL 
https://merenlab.org/m/anvi-estimate-metabolism serves a tutorial for this program which details the modes of usage and output file formats. The statistical approach for

805 enrichment analysis is defined elsewhere (Shaiber et al., 2020), but briefly it computes enrichment scores for functions (or metabolic modules) within groups by fitting a binomial generalized linear model (GLM) to the occurrence of each function or complete metabolic module in each group, and then computing a Rao test statistic, uncorrected p-values, and corrected q-values. We considered any function or metabolic module with a q-value less

810 than 0.05 to be 'enriched' in its associated group if it was also at least $75 \%$ complete and in at least $50 \%$ of the group members. To display the distribution of individual KEGG orthologs across genomes and order them based on their enrichment scores and group affiliations we used the program 'anvi-display-functions'.

\section{Determination of high-fitness and low-fitness MAGs for metabolic enrichment}

815 analysis. We classified MAGs as high-fitness if, in all 5 recipients, they were detected in the donor sample used for transplantation as well as the recipient more than 7 days postFMT. We classified low-fitness MAGs as those that, in at least 3 recipients, were detected in the donor sample used for FMT but were not detected in the recipient at least 7 days post-FMT. We reduced the number of high-fitness MAGs to be the same as the number

820 of low-fitness MAGs for metabolic enrichment analysis by selecting only the high-fitness MAGs which were the most prevalent in the Canadian gut metagenomes.

Ordination plots. We used the R vegan v2.4-2 package 'metaMDS' function to perform nonmetric multidimensional scaling (NMDS) with Horn-Morisita dissimilarity distance to compare taxonomic composition between donor, recipient, and global metagenomes. We 825 visualized ordination plots using $\mathrm{R}$ ggplot2.

\section{Code and Data Availability}

Raw sequencing data for donor and recipient metagenomes are stored under the NCBI BioProject PRJNA701961 (see Supplementary Table 1 for accession numbers for each sample). The URL https://merenlab.org/data/fmt-colonization serves reproducible 830 bioinformatics workflow and gives access to ad hoc scripts, usage instructions, and 
intermediate data objects to reproduce findings in our study. Supplementary tables are also accessible via doi:10.6084/m9.figshare.14138405.

\section{Acknowledgements}

We thank Mitchell L. Sogin, Eugene B. Chang, Samuel H. Light, and Howard A. Shuman

835 for helpful discussions, Ryan Moore and Ozcan C. Esen for technical support, and Nicola Segata and the members of the Segata group for their assistance with genomes from healthy gut metagenomes. We also thank Kaiyu Wu, Robyn Louie and Linda Ward of the IPC Research Laboratory at the University of Calgary for their help with patient recruitment and sampling. ARW was supported by the Robert C. and Mary Jane Gallo

840 Scholarship Fund. IV acknowledges support from the National Science Foundation Graduate Research Fellowship (1746045). This project was possible thanks to the generous support from the GI Research Foundation (GIRF) and the Mutchnik Family Fund.

\section{Author Contributions}

845 AME, TL, BJ conceived the study. JZD, MS, DK, TL recruited patients, performed transplantation experiments, and collected samples. ARW, JF, AME performed primary data analyses. IV developed research tools. KL, STML, HGM performed sample processing and sequencing. FT, AS, EF, JMR, CQ, MKY, AY contributed to data analyses and interpretation. DTR, BJ, TL, and AME directed research. ARW, JF, AME wrote the 850 paper with critical input from all authors.

\section{Competing Interests}

The authors declare no competing interests. 


\section{References}

Almeida, C., Oliveira, R., Soares, R., and Barata, P. (2020). Influence of gut microbiota dysbiosis on brain function: a systematic review. Porto Biomed J 5.

Alneberg, J., Bjarnason, B.S., de Bruijn, I., Schirmer, M., Quick, J., ljaz, U.Z., Lahti, L., Loman, N.J., Andersson, A.F., and Quince, C. (2014). Binning metagenomic contigs by coverage and composition. Nat. Methods 11, 1144-1146.

Altschul, S.F., Gish, W., Miller, W., Myers, E.W., and Lipman, D.J. (1990). Basic local 860 alignment search tool. J. Mol. Biol. 215, 403-410.

Aramaki, T., Blanc-Mathieu, R., Endo, H., Ohkubo, K., Kanehisa, M., Goto, S., and Ogata, H. (2020). KofamKOALA: KEGG Ortholog assignment based on profile HMM and adaptive score threshold. Bioinformatics 36, 2251-2252.

Arboleya, S., Watkins, C., Stanton, C., and Ross, R.P. (2016). Gut Bifidobacteria 865 Populations in Human Health and Aging. Front. Microbiol. 7, 1204.

Arumugam, M., Raes, J., Pelletier, E., Le Paslier, D., Yamada, T., Mende, D.R., Fernandes, G.R., Tap, J., Bruls, T., Batto, J.-M., et al. (2011). Enterotypes of the human gut microbiome. Nature 473, 174-180.

Bäckhed, F., Fraser, C.M., Ringel, Y., Sanders, M.E., Sartor, R.B., Sherman, P.M., 870 Versalovic, J., Young, V., and Finlay, B.B. (2012). Defining a healthy human gut microbiome: current concepts, future directions, and clinical applications. Cell Host Microbe 12, 611-622.

Barrangou, R., Briczinski, E.P., Traeger, L.L., Loquasto, J.R., Richards, M., Horvath, P., Coûté-Monvoisin, A.-C., Leyer, G., Rendulic, S., Steele, J.L., et al. (2009). Comparison 875 of the complete genome sequences of Bifidobacterium animalis subsp. lactis DSM 10140 and BI-04. J. Bacteriol. 191, 4144-4151.

Baumgart, D.C., and Carding, S.R. (2007). Inflammatory bowel disease: cause and immunobiology. Lancet 369, 1627-1640.

Biesalski, H.K. (2016). Nutrition meets the microbiome: micronutrients and the 880 microbiota. Ann. N. Y. Acad. Sci. 1372, 53-64.

Bowers, R.M., Kyrpides, N.C., Stepanauskas, R., Harmon-Smith, M., Doud, D., Reddy, T.B.K., Schulz, F., Jarett, J., Rivers, A.R., Eloe-Fadrosh, E.A., et al. (2017). Minimum information about a single amplified genome (MISAG) and a metagenome-assembled genome (MIMAG) of bacteria and archaea. Nat. Biotechnol. 35, 725-731.

885 Campbell, J.H., O’Donoghue, P., Campbell, A.G., Schwientek, P., Sczyrba, A., Woyke, T., Söll, D., and Podar, M. (2013). UGA is an additional glycine codon in uncultured SR1 bacteria from the human microbiota. Proc. Natl. Acad. Sci. U. S. A. 110, 5540-5545. 
Chaumeil, P.-A., Mussig, A.J., Hugenholtz, P., and Parks, D.H. (2019). GTDB-Tk: a toolkit to classify genomes with the Genome Taxonomy Database. Bioinformatics.

890 Chen, L.-X., Anantharaman, K., Shaiber, A., Eren, A.M., and Banfield, J.F. (2020). Accurate and complete genomes from metagenomes. Genome Res. 30, 315-333.

Chien, H.-L., Huang, H.-Y., and Chou, C.-C. (2006). Transformation of isoflavone phytoestrogens during the fermentation of soymilk with lactic acid bacteria and bifidobacteria. Food Microbiol. 23, 772-778.

895 Chow, J., Tang, H., and Mazmanian, S.K. (2011). Pathobionts of the gastrointestinal microbiota and inflammatory disease. Curr. Opin. Immunol. 23, 473-480.

Clooney, A.G., Eckenberger, J., Laserna-Mendieta, E., Sexton, K.A., Bernstein, M.T., Vagianos, K., Sargent, M., Ryan, F.J., Moran, C., Sheehan, D., et al. (2021). Ranking microbiome variance in inflammatory bowel disease: a large longitudinal intercontinental 900 study. Gut 70, 499-510.

Costello, E.K., Stagaman, K., Dethlefsen, L., Bohannan, B.J.M., and Relman, D.A. (2012). The application of ecological theory toward an understanding of the human microbiome. Science 336, 1255-1262.

D’Aimmo, M.R., Mattarelli, P., Biavati, B., Carlsson, N.G., and Andlid, T. (2012). The 905 potential of bifidobacteria as a source of natural folate. J. Appl. Microbiol. 112, 975-984.

David, L.A., Materna, A.C., Friedman, J., Campos-Baptista, M.I., Blackburn, M.C., Perrotta, A., Erdman, S.E., and Alm, E.J. (2014). Host lifestyle affects human microbiota on daily timescales. Genome Biol. 15, R89.

Delmont, T.O., and Eren, A.M. (2016). Identifying contamination with advanced

910 visualization and analysis practices: metagenomic approaches for eukaryotic genome assemblies. PeerJ 4, e1839.

Delmont, T.O., Quince, C., Shaiber, A., Esen, Ö.C., Lee, S.T., Rappé, M.S., McLellan, S.L., Lücker, S., and Eren, A.M. (2018). Nitrogen-fixing populations of Planctomycetes and Proteobacteria are abundant in surface ocean metagenomes. Nat Microbiol 3, 804915813.

Denef, V.J. (2019). Peering into the Genetic Makeup of Natural Microbial Populations Using Metagenomics. In Population Genomics: Microorganisms, M.F. Polz, and O.P. Rajora, eds. (Cham: Springer International Publishing), pp. 49-75.

De Preter, V., Bulteel, V., Suenaert, P., Geboes, K.P., De Hertogh, G., Luypaerts, A., 920 Geboes, K., Verbeke, K., and Rutgeerts, P. (2009). Pouchitis, similar to active ulcerative colitis, is associated with impaired butyrate oxidation by intestinal mucosa. Inflamm. Bowel Dis. 15, 335-340.

D’Souza, G., Shitut, S., Preussger, D., Yousif, G., Waschina, S., and Kost, C. (2018). 
925 Rep. 35, 455-488.

Durack, J., and Lynch, S.V. (2019). The gut microbiome: Relationships with disease and opportunities for therapy. J. Exp. Med. 216, 20-40.

Eddy, S.R. (2011). Accelerated Profile HMM Searches. PLoS Comput. Biol. 7, e1002195.

930 Edgar, R.C. (2004). MUSCLE: multiple sequence alignment with high accuracy and high throughput. Nucleic Acids Res. 32, 1792-1797.

Eiseman, B., Silen, W., Bascom, G.S., and Kauvar, A.J. (1958). Fecal enema as an adjunct in the treatment of pseudomembranous enterocolitis. Surgery 44, 854-859.

Eisenstein, M. (2020). The hunt for a healthy microbiome. Nature 577, S6-S8.

935 Enright, A.J., Van Dongen, S., and Ouzounis, C.A. (2002). An efficient algorithm for large-scale detection of protein families. Nucleic Acids Res. 30, 1575-1584.

Eren, A.M., Vineis, J.H., Morrison, H.G., and Sogin, M.L. (2013). A filtering method to generate high quality short reads using illumina paired-end technology. PLoS One 8 , e66643.

940 Eren, A.M., Esen, Ö.C., Quince, C., Vineis, J.H., Morrison, H.G., Sogin, M.L., and Delmont, T.O. (2015). Anvi'o: an advanced analysis and visualization platform for 'omics data. PeerJ 3, e1319.

Eren, A.M., Kiefl, E., Shaiber, A., Veseli, I., Miller, S.E., Schechter, M.S., Fink, I., Pan, J.N., Yousef, M., Fogarty, E.C., et al. (2021). Community-led, integrated, reproducible

945 multi-omics with anvi'o. Nat Microbiol 6, 3-6.

Feng, L., Raman, A.S., Hibberd, M.C., Cheng, J., Griffin, N.W., Peng, Y., Leyn, S.A., Rodionov, D.A., Osterman, A.L., and Gordon, J.I. (2020). Identifying determinants of bacterial fitness in a model of human gut microbial succession. Proc. Natl. Acad. Sci. U. S. A. $117,2622-2633$.

950 Finucane, M.M., Sharpton, T.J., Laurent, T.J., and Pollard, K.S. (2014). A taxonomic signature of obesity in the microbiome? Getting to the guts of the matter. PLoS One 9 , e84689.

Galperin, M.Y., Wolf, Y.I., Makarova, K.S., Vera Alvarez, R., Landsman, D., and Koonin, E.V. (2021). COG database update: focus on microbial diversity, model organisms, and 955 widespread pathogens. Nucleic Acids Res. 49, D274-D281.

Gomes, A.M.P., and Malcata, F.X. (1999). Bifidobacterium spp. and Lactobacillus acidophilus: biological, biochemical, technological and therapeutical properties relevant for use as probiotics. Trends Food Sci. Technol. 10, 139-157. 
Grehan, M.J., Borody, T.J., Leis, S.M., Campbell, J., Mitchell, H., and Wettstein, A.

960 (2010). Durable alteration of the colonic microbiota by the administration of donor fecal flora. J. Clin. Gastroenterol. 44, 551-561.

Herrmann, K.M., and Weaver, L.M. (1999). THE SHIKIMATE PATHWAY. Annu. Rev. Plant Physiol. Plant Mol. Biol. 50, 473-503.

Human Microbiome Project Consortium (2012). Structure, function and diversity of the 965 healthy human microbiome. Nature 486, 207-214.

Hyatt, D., Chen, G.-L., Locascio, P.F., Land, M.L., Larimer, F.W., and Hauser, L.J. (2010). Prodigal: prokaryotic gene recognition and translation initiation site identification. BMC Bioinformatics 11, 119.

Isaac, S., Scher, J.U., Djukovic, A., Jiménez, N., Littman, D.R., Abramson, S.B., Pamer, 970 E.G., and Ubeda, C. (2017). Short- and long-term effects of oral vancomycin on the human intestinal microbiota. J. Antimicrob. Chemother. 72, 128-136.

Joossens, M., Huys, G., Cnockaert, M., De Preter, V., Verbeke, K., Rutgeerts, P., Vandamme, P., and Vermeire, S. (2011). Dysbiosis of the faecal microbiota in patients with Crohn's disease and their unaffected relatives. Gut 60, 631-637.

975 Jungersen, M., Wind, A., Johansen, E., Christensen, J.E., Stuer-Lauridsen, B., and Eskesen, D. (2014). The Science behind the Probiotic Strain Bifidobacterium animalis subsp. lactis BB-12(®). Microorganisms 2, 92-110.

Kanehisa, M., and Goto, S. (2000). KEGG: kyoto encyclopedia of genes and genomes. Nucleic Acids Res. 28, 27-30.

980 Kanehisa, M., Goto, S., Sato, Y., Kawashima, M., Furumichi, M., and Tanabe, M. (2014). Data, information, knowledge and principle: back to metabolism in KEGG. Nucleic Acids Res. 42, D199-D205.

Kanehisa, M., Furumichi, M., Tanabe, M., Sato, Y., and Morishima, K. (2017). KEGG: new perspectives on genomes, pathways, diseases and drugs. Nucleic Acids Res. 45, 985 D353-D361.

Kao, D., Roach, B., Silva, M., Beck, P., Rioux, K., Kaplan, G.G., Chang, H.-J., Coward, S., Goodman, K.J., Xu, H., et al. (2017). Effect of Oral Capsule- vs ColonoscopyDelivered Fecal Microbiota Transplantation on Recurrent Clostridium difficile Infection: A Randomized Clinical Trial. JAMA 318, 1985-1993.

990 Khoruts, A., Dicksved, J., Jansson, J.K., and Sadowsky, M.J. (2010). Changes in the composition of the human fecal microbiome after bacteriotherapy for recurrent Clostridium difficile-associated diarrhea. J. Clin. Gastroenterol. 44, 354-360.

Kirchhelle, C. (2018). Pharming animals: a global history of antibiotics in food production (1935-2017). Palgrave Communications 4, 96. 
995 Kleerebezem, M., and Vaughan, E.E. (2009). Probiotic and gut lactobacilli and bifidobacteria: molecular approaches to study diversity and activity. Annu. Rev. Microbiol. 63, 269-290.

Koenig, J.E., Spor, A., Scalfone, N., Fricker, A.D., Stombaugh, J., Knight, R., Angenent, L.T., and Ley, R.E. (2011). Succession of microbial consortia in the developing infant 1000 gut microbiome. Proc. Natl. Acad. Sci. U. S. A. 108 Suppl 1, 4578-4585.

Köster, J., and Rahmann, S. (2012). Snakemake-a scalable bioinformatics workflow engine. Bioinformatics 28, 2520-2522.

Kowarsky, M., Camunas-Soler, J., Kertesz, M., De Vlaminck, I., Koh, W., Pan, W., Martin, L., Neff, N.F., Okamoto, J., Wong, R.J., et al. (2017). Numerous uncharacterized 1005 and highly divergent microbes which colonize humans are revealed by circulating cellfree DNA. Proc. Natl. Acad. Sci. U. S. A.

Langmead, B., and Salzberg, S.L. (2012). Fast gapped-read alignment with Bowtie 2. Nat. Methods 9, 357-359.

Lee, S.M., Donaldson, G.P., Mikulski, Z., Boyajian, S., Ley, K., and Mazmanian, S.K. 1010 (2013). Bacterial colonization factors control specificity and stability of the gut microbiota. Nature 501, 426-429.

Lee, S.T.M., Kahn, S.A., Delmont, T.O., Shaiber, A., Esen, Ö.C., Hubert, N.A., Morrison, H.G., Antonopoulos, D.A., Rubin, D.T., and Eren, A.M. (2017). Tracking microbial colonization in fecal microbiota transplantation experiments via genome1015 resolved metagenomics. Microbiome 5, 50.

Ley, R.E., Turnbaugh, P.J., Klein, S., and Gordon, J.I. (2006). Microbial ecology: human gut microbes associated with obesity. Nature 444, 1022-1023.

Li, H., Handsaker, B., Wysoker, A., Fennell, T., Ruan, J., Homer, N., Marth, G., Abecasis, G., Durbin, R., and 1000 Genome Project Data Processing Subgroup (2009).

1020 The Sequence Alignment/Map format and SAMtools. Bioinformatics 25, 2078-2079.

Lloyd-Price, J., Abu-Ali, G., and Huttenhower, C. (2016). The healthy human microbiome. Genome Med. 8, 51.

Lloyd-Price, J., Arze, C., Ananthakrishnan, A.N., Schirmer, M., Avila-Pacheco, J., Poon, T.W., Andrews, E., Ajami, N.J., Bonham, K.S., Brislawn, C.J., et al. (2019). Multi-omics 1025 of the gut microbial ecosystem in inflammatory bowel diseases. Nature 569, 655-662.

Lynch, S.V., and Pedersen, O. (2016). The Human Intestinal Microbiome in Health and Disease. N. Engl. J. Med. 375, 2369-2379.

Maignien, L., DeForce, E.A., Chafee, M.E., Eren, A.M., and Simmons, S.L. (2014). Ecological succession and stochastic variation in the assembly of Arabidopsis thaliana 1030 phyllosphere communities. MBio 5, e00682-13. 
Martens, J.H., Barg, H., Warren, M.J., and Jahn, D. (2002). Microbial production of vitamin B12. Appl. Microbiol. Biotechnol. 58, 275-285.

Martiny, A.C., Treseder, K., and Pusch, G. (2013). Phylogenetic conservatism of functional traits in microorganisms. ISME J. 7, 830-838.

1035 McBurney, M.I., Davis, C., Fraser, C.M., Schneeman, B.O., Huttenhower, C., Verbeke, K., Walter, J., and Latulippe, M.E. (2019). Establishing What Constitutes a Healthy Human Gut Microbiome: State of the Science, Regulatory Considerations, and Future Directions. J. Nutr. 149, 1882-1895.

1040 ecological forces that shape the bacterial communities of the human gut. Mucosal Immunol. 10, 567-579.

Minoche, A.E., Dohm, J.C., and Himmelbauer, H. (2011). Evaluation of genomic highthroughput sequencing data generated on Illumina HiSeq and genome analyzer systems. Genome Biol. 12, R112.

1045 Ott, S.J., Musfeldt, M., Wenderoth, D.F., Hampe, J., Brant, O., Fölsch, U.R., Timmis, K.N., and Schreiber, S. (2004). Reduction in diversity of the colonic mucosa associated bacterial microflora in patients with active inflammatory bowel disease. Gut 53, 685693.

Parks, D.H., Chuvochina, M., Waite, D.W., Rinke, C., Skarshewski, A., Chaumeil, P.-A., 1050 and Hugenholtz, P. (2018). A standardized bacterial taxonomy based on genome phylogeny substantially revises the tree of life. Nat. Biotechnol. 36, 996-1004.

Pasolli, E., Asnicar, F., Manara, S., Zolfo, M., Karcher, N., Armanini, F., Beghini, F., Manghi, P., Tett, A., Ghensi, P., et al. (2019). Extensive Unexplored Human Microbiome Diversity Revealed by Over 150,000 Genomes from Metagenomes Spanning Age, 1055 Geography, and Lifestyle. Cell 176, 649-662.e20.

Peng, Y., Leung, H.C.M., Yiu, S.M., and Chin, F.Y.L. (2012). IDBA-UD: a de novo assembler for single-cell and metagenomic sequencing data with highly uneven depth. Bioinformatics 28, 1420-1428.

Philippot, L., Andersson, S.G.E., Battin, T.J., Prosser, J.I., Schimel, J.P., Whitman, 1060 W.B., and Hallin, S. (2010). The ecological coherence of high bacterial taxonomic ranks. Nat. Rev. Microbiol. 8, 523-529.

Plichta, D.R., Graham, D.B., Subramanian, S., and Xavier, R.J. (2019). Therapeutic Opportunities in Inflammatory Bowel Disease: Mechanistic Dissection of HostMicrobiome Relationships. Cell 178, 1041-1056.

1065 Podlesny, D., and Florian Fricke, W. (2020). Microbial Strain Engraftment, Persistence and Replacement after Fecal Microbiota Transplantation. medRxiv 2020.09.29.20203638. 
Pompei, A., Cordisco, L., Amaretti, A., Zanoni, S., Matteuzzi, D., and Rossi, M. (2007). Folate production by bifidobacteria as a potential probiotic property. Appl. Environ.

1070 Microbiol. 73, 179-185.

Price, M.N., Dehal, P.S., and Arkin, A.P. (2010). FastTree 2--approximately maximumlikelihood trees for large alignments. PLoS One 5, e9490.

Pritchard, L., Glover, R.H., Humphris, S., Elphinstone, J.G., and Toth, I.K. (2016). Genomics and taxonomy in diagnostics for food security: soft-rotting enterobacterial 1075 plant pathogens. Anal. Methods 8, 12-24.

Quince, C., ljaz, U.Z., Loman, N., Eren, A.M., Saulnier, D., Russell, J., Haig, S.J., Calus, S.T., Quick, J., Barclay, A., et al. (2015). Extensive Modulation of the Fecal Metagenome in Children With Crohn's Disease During Exclusive Enteral Nutrition. Am. J. Gastroenterol. 110, 1718-1729; quiz 1730.

1080 Quince, C., Delmont, T.O., Raguideau, S., Alneberg, J., Darling, A.E., Collins, G., and Eren, A.M. (2017). DESMAN: a new tool for de novo extraction of strains from metagenomes. Genome Biol. 18, 181.

Rinke, C., Schwientek, P., Sczyrba, A., Ivanova, N.N., Anderson, I.J., Cheng, J.-F., Darling, A., Malfatti, S., Swan, B.K., Gies, E.A., et al. (2013). Insights into the phylogeny 1085 and coding potential of microbial dark matter. Nature 499, 431-437.

Rothschild, D., Weissbrod, O., Barkan, E., Kurilshikov, A., Korem, T., Zeevi, D., Costea, P.I., Godneva, A., Kalka, I.N., Bar, N., et al. (2018). Environment dominates over host genetics in shaping human gut microbiota. Nature 555, 210-215.

Schell, M.A., Karmirantzou, M., Snel, B., Vilanova, D., Berger, B., Pessi, G., Zwahlen, 1090 M.-C., Desiere, F., Bork, P., Delley, M., et al. (2002). The genome sequence of Bifidobacterium longum reflects its adaptation to the human gastrointestinal tract. Proc. Natl. Acad. Sci. U. S. A. 99, 14422-14427.

Schirmer, M., Garner, A., Vlamakis, H., and Xavier, R.J. (2019). Microbial genes and pathways in inflammatory bowel disease. Nat. Rev. Microbiol. 17, 497-511.

1095 Schmidt, T.S.B., Raes, J., and Bork, P. (2018). The Human Gut Microbiome: From Association to Modulation. Cell 172, 1198-1215.

Shahinas, D., Silverman, M., Sittler, T., Chiu, C., Kim, P., Allen-Vercoe, E., Weese, S., Wong, A., Low, D.E., and Pillai, D.R. (2012). Toward an understanding of changes in diversity associated with fecal microbiome transplantation based on 16S rRNA gene 1100 deep sequencing. MBio 3, e00338-12.

Shaiber, A., Willis, A.D., Delmont, T.O., Roux, S., Chen, L.-X., Schmid, A.C., Yousef, M., Watson, A.R., Lolans, K., Esen, Ö.C., et al. (2020). Functional and genetic markers of niche partitioning among enigmatic members of the human oral microbiome. Genome Biol. 21, 292. 
1105 Sharon, I., Morowitz, M.J., Thomas, B.C., Costello, E.K., Relman, D.A., and Banfield, J.F. (2013). Time series community genomics analysis reveals rapid shifts in bacterial species, strains, and phage during infant gut colonization. Genome Res. 23, 111-120.

Sheth, R.U., Li, M., Jiang, W., Sims, P.A., Leong, K.W., and Wang, H.H. (2019). Spatial metagenomic characterization of microbial biogeography in the gut. Nat. Biotechnol. 37,

1110 877-883.

Smillie, C.S., Sauk, J., Gevers, D., Friedman, J., Sung, J., Youngster, I., Hohmann, E.L., Staley, C., Khoruts, A., Sadowsky, M.J., et al. (2018). Strain Tracking Reveals the Determinants of Bacterial Engraftment in the Human Gut Following Fecal Microbiota Transplantation. Cell Host Microbe 23, 229-240.e5.

1115 Sokol, H., and Seksik, P. (2010). The intestinal microbiota in inflammatory bowel diseases: time to connect with the host. Curr. Opin. Gastroenterol. 26, 327-331.

Strozzi, G.P., and Mogna, L. (2008). Quantification of folic acid in human feces after administration of Bifidobacterium probiotic strains. J. Clin. Gastroenterol. 42 Suppl 3 Pt 2, S179-S184.

1120 Sugahara, H., Odamaki, T., Hashikura, N., Abe, F., and Xiao, J.-Z. (2015). Differences in folate production by bifidobacteria of different origins. Biosci Microbiota Food Health 34, 87-93.

Swidsinski, A., Weber, J., Loening-Baucke, V., Hale, L.P., and Lochs, H. (2005). Spatial organization and composition of the mucosal flora in patients with inflammatory bowel

1125 disease. J. Clin. Microbiol. 43, 3380-3389.

Tatusov, R.L., Fedorova, N.D., Jackson, J.D., Jacobs, A.R., Kiryutin, B., Koonin, E.V., Krylov, D.M., Mazumder, R., Mekhedov, S.L., Nikolskaya, A.N., et al. (2003). The COG database: an updated version includes eukaryotes. BMC Bioinformatics 4, 41.

Utter, D.R., Borisy, G.G., Eren, A.M., Cavanaugh, C.M., and Mark Welch, J.L. (2020).

1130 Metapangenomics of the oral microbiome provides insights into habitat adaptation and cultivar diversity. Genome Biol. 21, 293.

Vanni, C., Schechter, M.S., Acinas, S.G., Barberán, A., Buttigieg, P.L., Casamayor, E.O., Delmont, T.O., Duarte, C.M., Murat Eren, A., Finn, R.D., et al. (2020). Unifying the global coding sequence space enables the study of genes with unknown function

1135 across biomes.

Ventura, M., O’Flaherty, S., Claesson, M.J., Turroni, F., Klaenhammer, T.R., van Sinderen, D., and O'Toole, P.W. (2009). Genome-scale analyses of health-promoting bacteria: probiogenomics. Nat. Rev. Microbiol. 7, 61-71.

Vineis, J.H., Ringus, D.L., Morrison, H.G., Delmont, T.O., Dalal, S., Raffals, L.H.,

1140 Antonopoulos, D.A., Rubin, D.T., Eren, A.M., Chang, E.B., et al. (2016). Patient-Specific Bacteroides Genome Variants in Pouchitis. MBio 7, e01713-e01716, 
1145 Associated Rodents. Cell 180, 221-232.

Wexler, A.G., and Goodman, A.L. (2017). An insider's perspective: Bacteroides as a window into the microbiome. Nature Microbiology 2.

Wood, D.E., Lu, J., and Langmead, B. (2019). Improved metagenomic analysis with Kraken 2. Genome Biol. 20, 257.

1150 Wu, G., Zhao, N., Zhang, C., Lam, Y.Y., and Zhao, L. (2021). Guild-based analysis for understanding gut microbiome in human health and diseases. Genome Med. 13, 22.

Yasuda, K., Oh, K., Ren, B., Tickle, T.L., Franzosa, E.A., Wachtman, L.M., Miller, A.D., Westmoreland, S.V., Mansfield, K.G., Vallender, E.J., et al. (2015). Biogeography of the intestinal mucosal and lumenal microbiome in the rhesus macaque. Cell Host Microbe

1155 17, 385-391.

\section{Supplementary Figures}
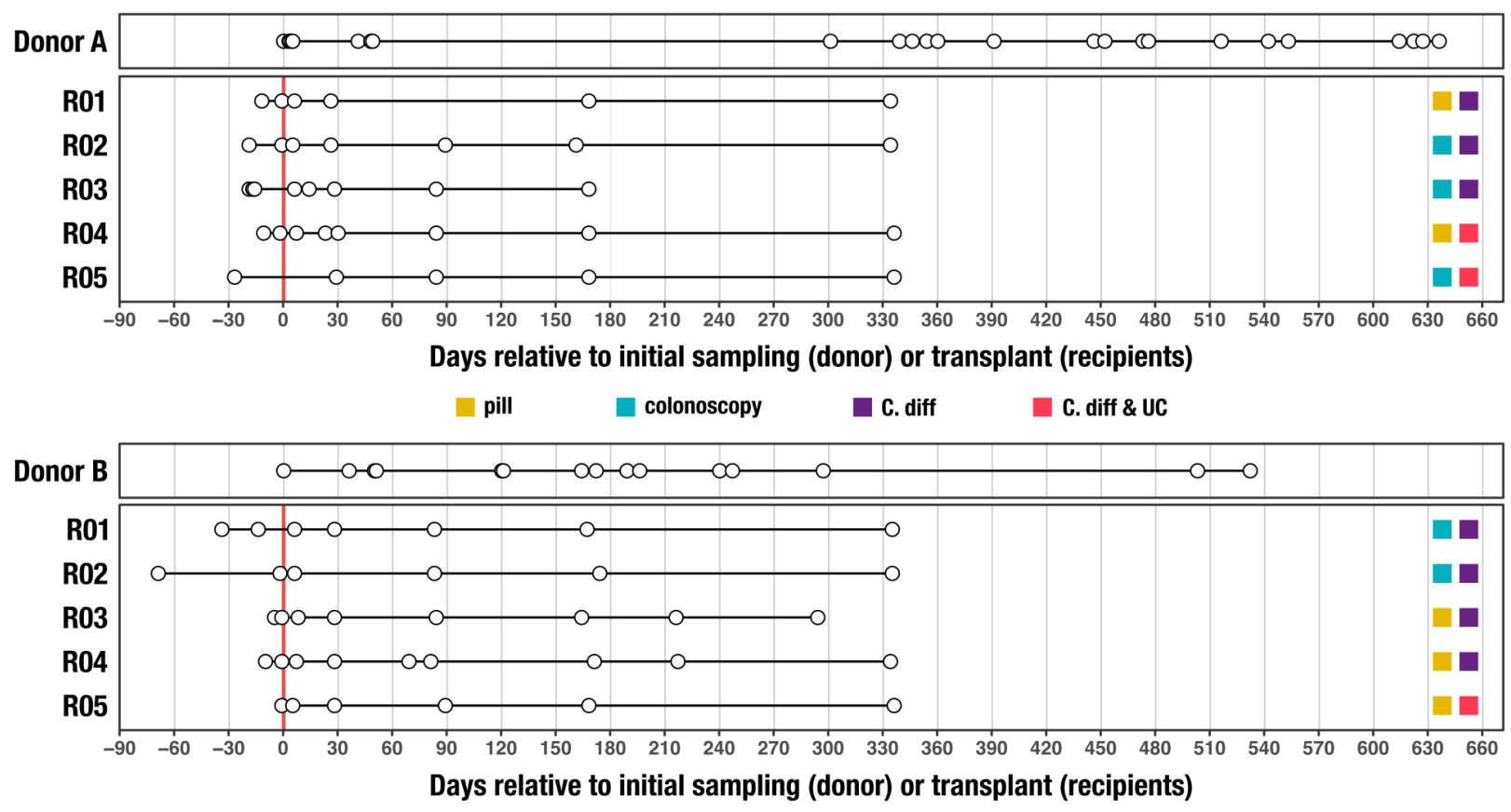

Supplementary Figure 1. Timeline of stool samples collected from FMT study. Each circle represents a stool sample collected from either an FMT donor or FMT recipient. The thicker, red vertical line at day 0 represents the FMT

1160 event for each recipient. FMT method (pill or colonoscopy) and FMT recipient health and disease state (C. diff - chronic recurrent Clostridium difficile infection, UC - ulcerative colitis) are indicated on the right. 
bioRxiv preprint doi: https://doi.org/10.1101/2021.03.02.433653; this version posted April 22, 2021. The copyright holder for this preprint (which was not certified by peer review) is the author/funder, who has granted bioRxiv a license to display the preprint in perpetuity. It is made available under aCC-BY-ND 4.0 International license.

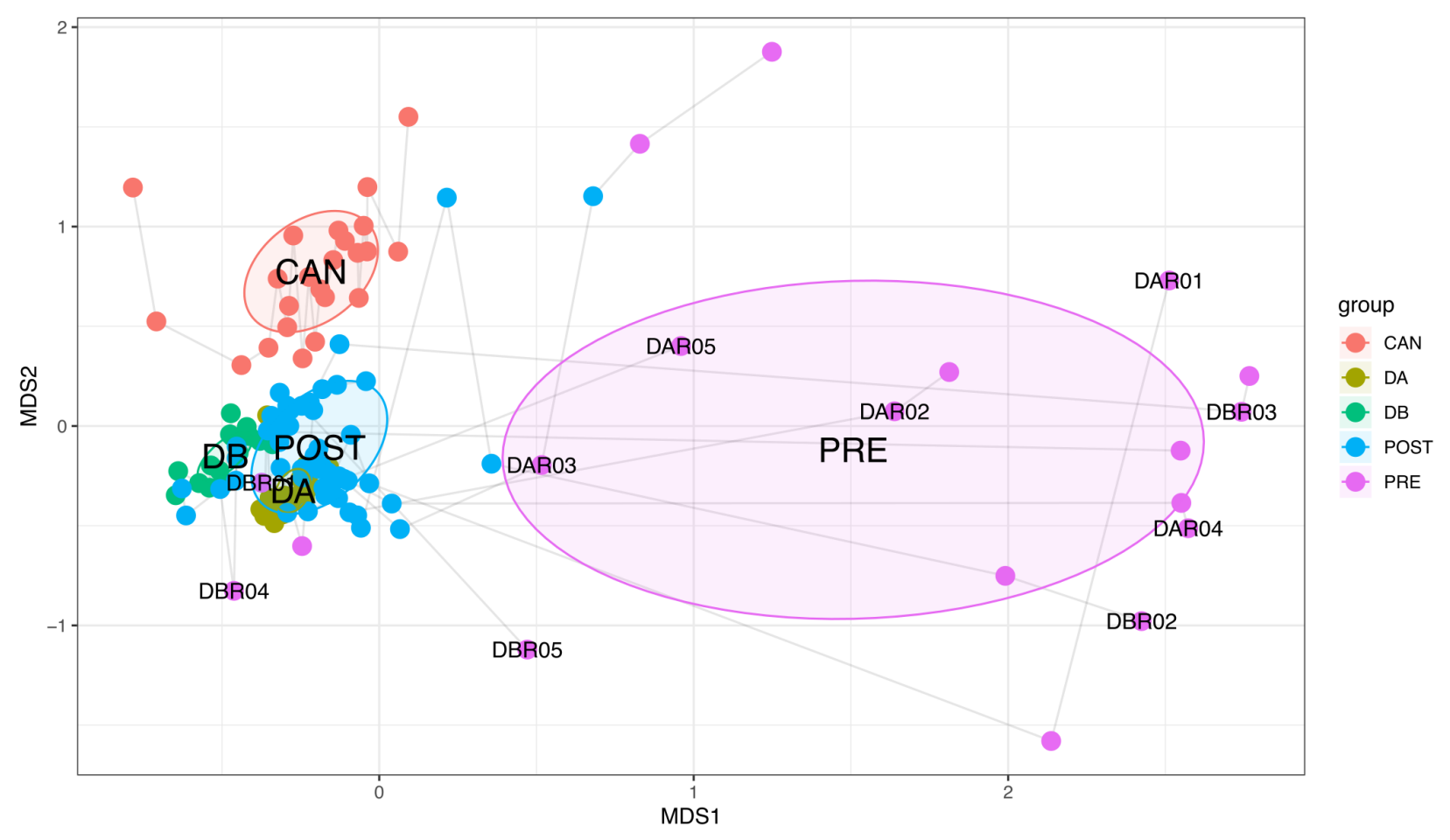

Supplementary Figure 2. Nonmetric multidimensional scaling (NMDS) ordination of the taxonomic composition of donor, recipient, and Canadian gut metagenomes at the genus level based on Morisita-Horn dissimilarity. Samples from the same participant are joined by lines with the earliest time point labeled. CAN: Canadian gut metagenomes, DA: donor A, DB: donor B, POST: recipients post-FMT, PRE: recipients pre-FMT. 
bioRxiv preprint doi: https://doi.org/10.1101/2021.03.02.433653; this version posted April 22, 2021. The copyright holder for this preprint (which was not certified by peer review) is the author/funder, who has granted bioRxiv a license to display the preprint in perpetuity. It is made available under aCC-BY-ND 4.0 International license.

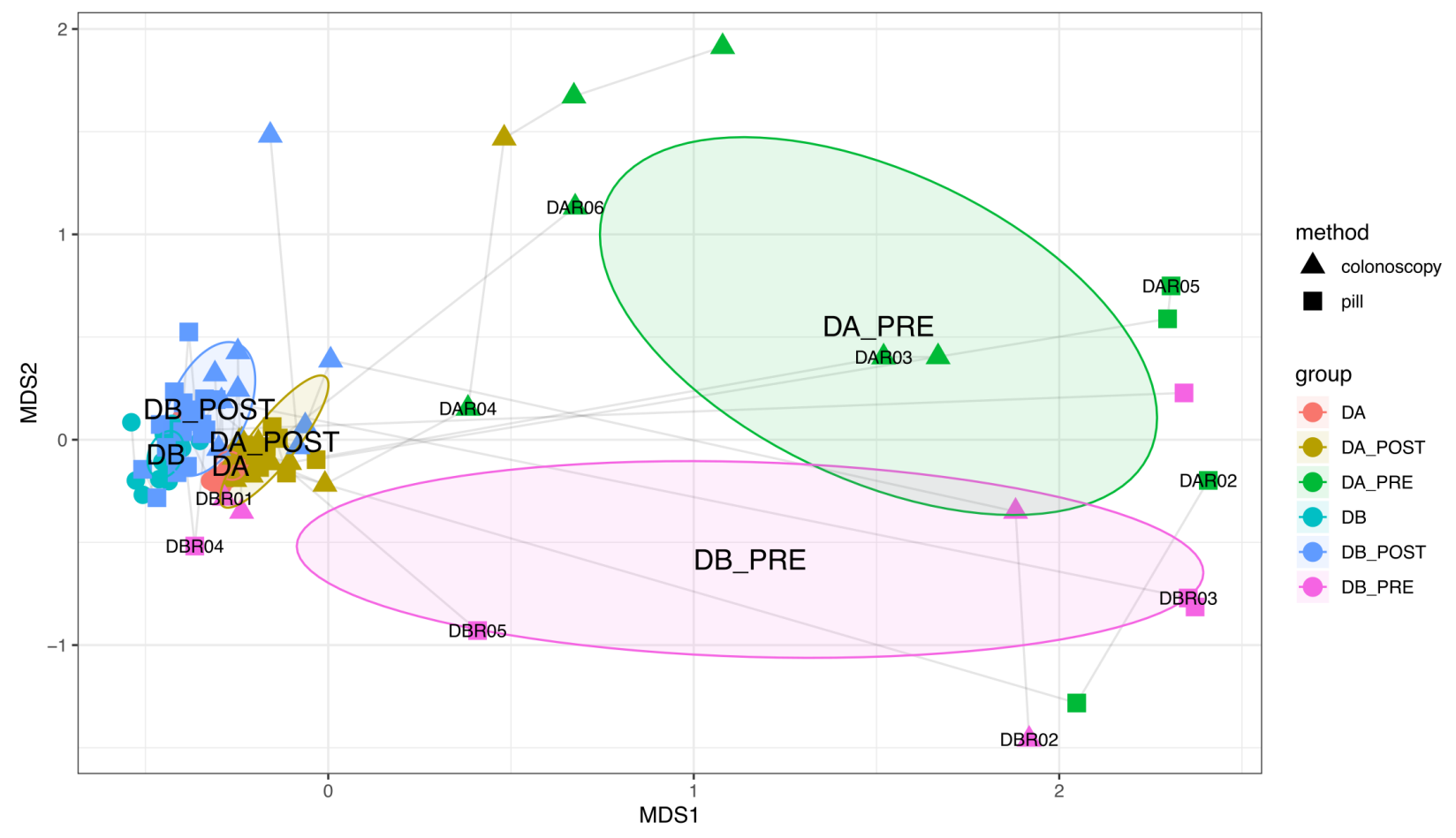

Supplementary Figure 3. Nonmetric multidimensional scaling (NMDS) ordination of the taxonomic composition of the donor and recipient metagenomes at genus level based on Morisita-Horn dissimilarity. Samples from the same participant are joined by lines with the earliest time point labeled. DA_POST: donor A recipients post-FMT, DA_PRE: donor A recipients pre-FMT, DA: donor A, DB_POST: donor B recipients post-FMT, DB_PRE: donor B recipients pre-FMT, DB: donor $B$. 
bioRxiv preprint doi: https://doi.org/10.1101/2021.03.02.433653; this version posted April 22, 2021. The copyright holder for this preprint (which was not certified by peer review) is the author/funder, who has granted bioRxiv a license to display the preprint in perpetuity. It is made available under aCC-BY-ND 4.0 International license.

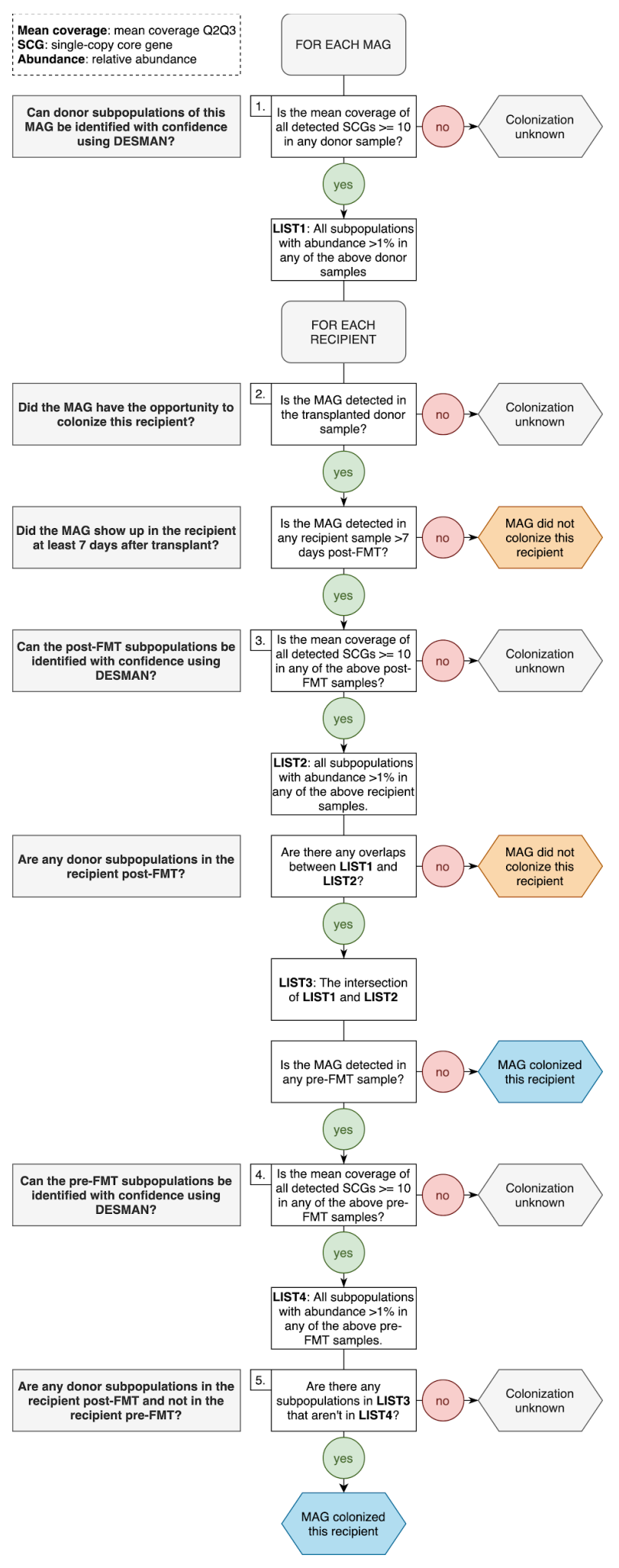

Supplementary Figure 4. A flowchart outlining our method to assign successful colonization, failed colonization, or 1175 undetermined colonization phenotypes to donor-derived populations in the recipients of that donor's stool. 


\section{Supplementary Tables ( $(2)$}

Supplementary Table 1: Description of FMT study and stool samples collected. a) Description of FMT donor stool samples and SRA accession numbers. b) Description of FMT recipient samples and SRA accession numbers. c) Description of transplantation events.

1180 Supplementary Table 2: Description of FMT metagenomes and co-assemblies. a) Metagenome SRA accession numbers and number of metagenomic short-reads sequenced and mapped to co-assemblies and MAGs. b) Phylum level taxonomic composition of metagenomes. c) Genus level taxonomic composition of metagenomes. d) Summary statistics for contigs from metagenome co-assemblies.

Supplementary Table 3: Description of MAGs. a) Summary statistics and taxonomic assignments for MAGs. b) and

1185 c) Detection of Donor A and Donor B MAGs in FMT metagenomes, respectively. d) and e) Detection of Donor A and Donor B MAGs in global gut metagenomes, respectively. $f$ ) and g) Detection summary statistics of Donor $A$ and Donor $B$ MAGs in global gut metagenomes, respectively. h) and i) Mean non-outlier coverage of Donor A and Donor B MAG single-copy core genes in FMT metagenomes.

Supplementary Table 4: Accession numbers of gut metagenomes from 17 countries.

1190 Supplementary Table 5: MAG subpopulation information. a) and b) Number of Donor A and Donor B MAG subpopulations detected in FMT metagenomes, respectively. c) and d) Subpopulation composition of Donor A and Donor B MAGs in FMT metagenomes, respectively.

Supplementary Table 6: MAG/recipient pair colonization outcomes and MAG mean coverage in the 2nd and 3rd quartiles in stool samples used for transplantation.

1195 Supplementary Table 7: Description of high vs. low-fitness populations. a) Taxonomic assignments and genome size estimates for high- and low-fitness populations. b) KEGG module completeness information for high- and lowfitness populations. c) Raw KEGG module enrichment information for high- and low-fitness populations. d) KEGG module enrichment and categorical information for the 33 modules enriched in high-fitness populations. e) and f) Completeness information for the 33 modules enriched in high-fitness populations in all high- and low-fitness

1200 populations.

Supplementary Table 8: a) List of genomes from healthy individuals and individuals with IBD. b) Module completion values across genomes.

Supplementary Table 9: Bifidobacteria functional analysis. a) Accession numbers for Bifidobacteria reference genomes. b) Summary statistics for Bifidobacteria MAGs and reference genomes. c) Prevalence of Bifidobacteria

1205 MAGs in global gut metagenomes. d) gANI percent identity between Bifidobacteria genomes. e) gANI percent alignment coverage between Bifidobacteria genomes. f) KOfams enriched in different Bifidobacteria species. g) KOfam presence and absence in Bifidobacteria genomes. h) COG functions enriched in different Bifidobacteria species. i) COG function presence and absence in Bifidobacteria genomes. j) KEGG modules enriched in different Bifidobacteria species. k) KEGG module completeness in Bifidobacteria genomes. 